\title{
Porcine Liver Carboxylesterase Requires Polyisoprenylation for High Affinity Binding to Cysteinyl Substrates
}

\author{
Nazarius S. Lamango ${ }^{1, *}$, Randolph Duverna ${ }^{1}$, Wang Zhang $^{1}$ and Seth Y. Ablordeppey ${ }^{1}$ \\ ${ }^{I}$ College of Pharmacy and Pharmaceutical Sciences, Florida A\&M University, Tallahassee, FL 32307, USA
}

\begin{abstract}
The polyisoprenylation pathway enzymes have been the focus of numerous studies to better understand the roles of polyisoprenylated proteins in eukaryotic cells and to identify novel targets against diseases such as cancer. The final step of the pathway is a reversible reaction catalyzed by isoprenyl carboxylmethyl transferase (icmt) whose products are then hydrolyzed by polyisoprenylated methylated protein methyl esterase (PMPMEase). Unlike the other pathway enzymes, the esterase has received little attention. We recently purified PMPMEase from porcine liver using an S-polyisoprenylated cysteine methyl ester substrate-dependent screening assay. However, no data is available showing its relative interaction with structurally diverse substrates. As such, its role as the putative endogenous PMPMEase has not been demonstrated. A series of substrates with S-alkyl substituents ranging from 2 to 20 carbons, including the two moieties found in polyisoprenylated proteins, were synthesized. Enzyme kinetics analysis revealed a 33-fold increase in affinity $\left(K_{\mathrm{M}}\right.$ values) from ethyl- $(\mathrm{C}-2,505 \pm 63 \mu \mathrm{M})$, prenyl- $(\mathrm{C}-5,294 \pm 25 \mu \mathrm{M})$, trans-geranyl- $(\mathrm{C}-10,87 \pm 12 \mu \mathrm{M})$, trans, trans-farnesyl- $(\mathrm{C}-15,29 \pm 2.2 \mu \mathrm{M})$ to all trans-geranylgeranyl- $(\mathrm{C}-20-, 15 \pm 2.7 \mu \mathrm{M})$ based analogs. Comparative molecular field analysis of the data yielded a cross-validated $\mathrm{q}^{2}$ of $0.863 \pm 0.365$ and a final $\mathrm{R}^{2}$ of 0.995 . Since the substrates with the S-trans, trans-farnesyl and S-all trans-geranylgeranyl moieties that occur in proteins show the highest affinity towards PMPMEase and are not hydrolyzed by the cholinesterases, the results suggest that polyisoprenylated proteins are the endogenous substrates of this esterase. The results suggest design strategies for high affinity and selective inhibitors of PMPMEase.
\end{abstract}

Keywords: Polyisoprenylation, Isoprenylation, Esterase, Carboxylesterase, Farnesyl, Geranylgeranyl, Farnesylation, Geranylgeranylation.

\section{INTRODUCTION}

Polyisoprenylation is an essential secondary modification on a wide range of proteins in eukaryotic cells [1,2]. Their contributions to disease due to mutations or improper metabolism have been widely reported [1]. For example, an estimated 15 to $30 \%$ of cancers involve overexpression [3] or mutations of monomeric G-proteins that render them constitutively active [4]. On the other extreme of the cell viability spectrum is the degeneration observed in choroideramia caused by a defective polyisoprenylation enzyme $[5,6]$. Heart-specific knockout mice devoid of the polyisoprenylated-dependent endoprotease eventually develop cardiomyopathy [7] while knockout mice lacking isoprenylated carboxylmethyltransferase (icmt, also known as prenylated protein methyl transferase, PPMTase) activity do not survive through mid gestation [8].

While the pathophysiological effects of deficient polyisoprenyl transferase and PPMTase activities yield critical insights into the importance of the polyisoprenylation pathway, the role of the esterase that counterbalances the effects of PPMTase and makes possible the only reversible step of the pathway is less understood. Polyisoprenyl binding sites

*Address correspondence to this author at the College of Pharmacy and Pharmaceutical Sciences, Florida A\&M University, Tallahassee, FL 32307; USA; Tel: 850412 7377; Lab: 850599 8443; Fax: 850599 3347;

E-mail: nazarius.lamango@famu.edu have been identified in some proteins, thus underlying the likelihood that polyisoprenylation promotes protein-protein interactions $[9,10]$. Given that PPMTase removes and PMPMEase introduces a negative charge in the vicinity of the polyisoprenyl moiety, it has been suggested that these enzymes switch the polyisoprenylated proteins between the active and inactive conformations [11].

Although the existence of PMPMEase has been predicted from biochemical studies [12, 13], few studies have been conducted to better understand its substrate specificity and tissue variants. PMPMEase was recently purified and identified from porcine liver [14]. It is over 70\% identical and $80 \%$ similar to various ubiquitously distributed carboxylesterases [14]. The crystal structures of human carboxylesterase 1 have been solved [15-18]. The data reveal an overarching feature of human carboxylesterase 1; that of a large, hydrophobic and flexible active site believed to accommodate a wide variety of substrates [15]. This may be an adaptation to accommodate the large and structurally diverse polypeptide portions bearing the hydrophobic polyisoprenylated methylated cysteines at their carboxyl terminals [14]. The present study is aimed at determining whether the enzyme is selective for polyisoprenylated methylated protein methyl esterase (PMPMEase), in which case the farnesylated and geranylgeranylated substrates would have the highest affinities for the enzyme. A series of S-alkyl,N-acyl cysteine methyl esters were thus synthesized, purified and characterized by proton and ${ }^{13} \mathrm{C}$ NMR and elemental analysis. Substrate kinetics analysis with the enzyme revealed a strong 
dependence on the polyisoprenyl moiety for high affinity binding to the esterase as shown by the significantly lower Michaelis-Menten constants for the S-trans,trans-farnesyl (C15) and S-all trans-geranylgeranyl (C20) compared to the S-ethyl cysteine methyl and other ester substrates. The requirement of polyisoprenylation for high affinity binding to PMPMEase and selectivity against cholinesterase hydrolysis is indicative of an endogenous role in the metabolism of polyisoprenylated proteins and important for the rational design of selective high-affinity inhibitors of the enzyme.

\section{MATERIALS AND METHODS}

\section{Materials}

Esterase from porcine liver (EPL), acetylcholinesterase (AChE) and butyrylcholinesterase (BChE) were obtained from Sigma-Aldrich, St. Louis, MO. SDS-PAGE electrophoresis gels were obtained from BioRad, Hercules, CA. SDSPAGE molecular weight markers were from Fisher Scientific (Suwanee, GA).

\section{Synthesis of Substrates}

Experiments were conducted under argon in flame-dried glassware and anhydrous solvents, when necessary (Scheme 1). Flash chromatography was performed on Fisher 40-63u silica gel. Unless otherwise stated, NMR spectra (300 MHz) were obtained in $\mathrm{CDCl}_{3}$ on Varian Mercury 300. Chemical shifts $(\delta)$ are given in $\mathrm{ppm}$ relative to the signal of the deuterated solvent. IR spectra were taken on Shimadzu 840S in $\mathrm{KBr}$ pellets. Optical rotations were measured on JASCO $\mathrm{P}-1020$ polarimeter in a $10 \mathrm{~cm}$ cell. All substrates were $>99 \%$ pure by HPLC. Elemental analyses were conducted by Atlantic Microlab, Inc, Norcross, GA, and the accepted values are within $0.4 \%$ of the theoretical values except otherwise noted.

trans-Geranyl bromide. A solution of $\mathrm{PBr}_{3}(6 \mathrm{ml}, 63.2$ $\mathrm{mmol})$ in anhydrous ether $(10 \mathrm{~mL})$ was added drop-wise to trans-geraniol $(21.7 \mathrm{~g}, 141 \mathrm{mmol})$ in anhydrous ether $(250 \mathrm{~mL})$ at -15 to $-10^{\circ} \mathrm{C}$. At the end of the addition, the mixture was kept at $0^{\circ} \mathrm{C}$ for $10 \mathrm{~min}$ and then at $20^{\circ} \mathrm{C}$ for 30 $\min$ [19]. The organic phase was decanted, washed with cold $5 \% \mathrm{NaHCO}_{3}$, brine, dried over $\mathrm{MgSO}_{4}$ and the solvent removed at $40^{\circ} \mathrm{C} / 3 \mathrm{~mm} \mathrm{Hg}$, giving a colorless liquid $(22.2 \mathrm{~g}$, 73\%). ${ }^{1} \mathrm{H}$ NMR: 1.60 (s, $\left.\mathrm{CH}_{3}\right), 1.69\left(\mathrm{~s}, \mathrm{CH}_{3}\right), 1.74\left(\mathrm{~s}, \mathrm{CH}_{3}\right)$,
1.95-2.20 (m, $\left.2 \mathrm{CH}_{2}\right), 4.03\left(\mathrm{~d}, \mathrm{CH}_{2} \mathrm{Br}, J=8.4 \mathrm{~Hz}\right), 5.09$ $(\mathrm{m}, \mathrm{C}=\mathrm{C} \underline{\mathrm{H}}), 5.54(\mathrm{~m}, \mathrm{C}=\mathrm{C} \underline{\mathrm{HCBr}}) ;{ }^{13} \mathrm{C}$ NMR: $16.2,17.9$, 25.9, 26.4, 29.9, 39.8, 120.8, 123.8, 132.2, 143.8.

trans, trans-Farnesyl bromide. A solution of $\mathrm{PBr}_{3}(6 \mathrm{~mL}$, $63.2 \mathrm{mmol})$ in anhydrous ether $(10 \mathrm{~mL})$ was added drop-wise to trans, trans-farnesol (20.5 g, $92 \mathrm{mmol})$ in anhydrous ether $(250 \mathrm{~mL})$ at -15 to $-10^{\circ} \mathrm{C}$ and treated as for the synthesis of trans-geranyl bromide. A pale-yellow liquid $(24.43 \mathrm{~g}, 93 \%)$ was obtained. ${ }^{1} \mathrm{H}$ NMR: $1.60\left(\mathrm{~s}, 2 \mathrm{CH}_{3}\right), 1.67\left(\mathrm{~s}, \mathrm{CH}_{3}\right), 1.72$ (s, $\left.\mathrm{CH}_{3}\right), 1.95-2.15\left(\mathrm{~m}, 4 \mathrm{CH}_{2}\right), 4.16\left(\mathrm{~d}, \mathrm{CH}_{2} \mathrm{Br}, J=8.4 \mathrm{~Hz}\right)$, 5.09 (m, $2 \times \mathrm{C}=\mathrm{CH}), 5.54$ (m, $\mathrm{C}=\mathrm{CHCBr}) ;{ }^{13} \mathrm{C}$ NMR: 16.2, $16.3,17.9,25.9,26.3,26.9,29.7,39.7,39.9,120.8,123.6$, $124.5,131.5,135.8,143.7$.

trans, trans, trans-Geranylgeranyl bromide: As for trans, trans-farnesyl bromide from $\mathrm{PBr}_{3}(1 \mathrm{~mL}, 1.1 \mathrm{mmol})$ and trans, trans, trans-geranylgeraniol $(1 \mathrm{~g}, 3.4 \mathrm{mmol})$ in anhydrous ether. A pale-yellow liquid $(1.2 \mathrm{~g})$ was obtained. ${ }^{1} \mathrm{H}$ NMR: 1.60 (s, $\left.3 \mathrm{CH}_{3}\right), 1.68\left(\mathrm{~s}, \mathrm{CH}_{3}\right), 1.73\left(\mathrm{~s}, \mathrm{CH}_{3}\right), 1.95$ $2.15\left(\mathrm{~m}, 6 \mathrm{CH}_{2}\right), 4.02\left(\mathrm{~d}, \mathrm{CH}_{2} \mathrm{Br}, J=8.1 \mathrm{~Hz}\right), 5.09(\mathrm{~m}, 3$ $\mathrm{C}=\mathrm{C} \underline{\mathrm{H}}), 5.53$ (m, $\mathrm{C}=\mathrm{C} \underline{\mathrm{HCBr}}) ;{ }^{13} \mathrm{C}$ NMR: $16.19,16.23,16.28$ $17.91,25.92,26.35,26.83,27.01,29.80,39.76,39.90,39.96$, $120.80,123.63,124.41,124.62,131.44,135.17,135.86$, 143.78 .

$(S)$-(+)-Citronellyl iodide. $\mathrm{PPh}_{3}(6.3 \mathrm{~g}, 24 \mathrm{mmol})$ was added to dry DCM $(80 \mathrm{ml})$ followed by imidazole $(1.64 \mathrm{~g}$, $24 \mathrm{mmol})$, iodine $(6.1 \mathrm{~g}, 24 \mathrm{mmol})$ and a solution of $(S)-(-)-$ citronellol $(3.6 \mathrm{ml}, 20 \mathrm{mmol})$ in dry DCM $(20 \mathrm{ml})$. The mixture was stirred at RT until the alcohol disappeared (TLC in DCM, $\mathrm{R}_{\mathrm{f}} 0.1$ alcohol, 0.9 iodide). The precipitate was filtered off, the solvent removed and the semi-solid residue was treated with hexane. The hexane extract was purified by column chromatography (in hexane) [20]. The solvent was removed under vacuum, yielding $5.1 \mathrm{~g}(96 \%)$ of a colorless liquid, $[\alpha]_{\mathrm{D}}{ }^{23}=+1.02^{\circ}$ (pure, $\left.1 \mathrm{~cm}\right) .{ }^{1} \mathrm{H}-\mathrm{NMR}$ : $\delta 0.88(\mathrm{~d}, 6.6$ $\left.\mathrm{Hz}, \mathrm{CHCH}_{3}\right), 1.19\left(\mathrm{~m}, \mathrm{H}_{4}\right), 1.33\left(\mathrm{~m}, \mathrm{H}_{4}\right), 1.5-1.7\left(\mathrm{~m}, \mathrm{H}_{2}+\right.$ $\left.\mathrm{C}_{\mathrm{HCH}_{3}}, 2 \mathrm{H}\right), 1.60\left(\mathrm{~s}, \mathrm{CH}_{3}\right), 1.68\left(\mathrm{~s}, \mathrm{CH}_{3}\right), 1.8-2.1\left(\mathrm{~m}, \mathrm{H}_{5,5}\right.$, $\left.+\mathrm{H}_{2}, 3 \mathrm{H}\right), 3.20\left(\mathrm{~m}, \mathrm{CH}_{2} \mathrm{I}\right), 5.08(\mathrm{~m}, \mathrm{C}=\mathrm{CH}) .{ }^{13} \mathrm{C}-\mathrm{NMR}: \delta$ $5.28,17.93,18.90,25.55,25.96,33.80,36.54,41.15,124.70$, 131.62 .

$(R)$-(-)-Citronellyl iodide. $(R)-(+)$-citronellal $(9.06 \mathrm{~mL}$, $50 \mathrm{mmol}$ ) was reduced with $1 \mathrm{~N}$ solution of $\mathrm{LiAlH}_{4}$ in ether $(55 \mathrm{ml}, 55 \mathrm{mmol})$. TLC control in hexane-EtOAc $4: 1, \mathrm{R}_{\mathrm{f}}$ of
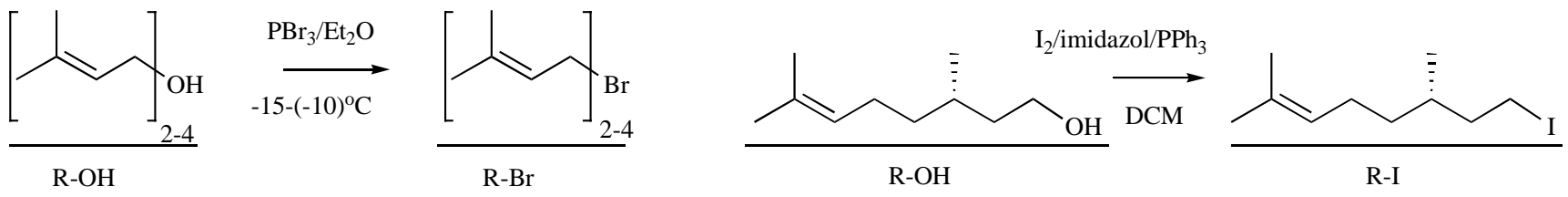<smiles>[R9]C[C@H](N)C(=O)OCCO</smiles><smiles>CC(=O)OOC(C)=O</smiles><smiles>[R9]C[C@H](NC(=O)c1ccc([N+](=O)[O-])cc1)C(=O)O[R]</smiles>

Scheme 1. General procedures for the synthesis of the S-alkylated N-acylated cysteine ester substrates. 
alcohol 0.2 , of aldehyde 0.5 . Vacuum distillation of the crude product $(6.1 \mathrm{~g}, 78 \%)$ resulted in $4.1 \mathrm{~g}$ of $(R)-(+)-$ citronellol, b.p. $85-87^{\circ} \mathrm{C} / 4 \mathrm{~mm} \mathrm{Hg},[\alpha]_{\mathrm{D}}{ }^{23.5}=+3.44^{\circ}$ (pure, 1 $\mathrm{cm})$. (R)-(+)-citronellol was converted to the iodide as described for the synthesis of (S)-(+)-Citronellyl iodide [21]. Instead of filtration, the solvent was removed under reduced pressure and the residue treated with 2 portions of hexane. Column chromatography of the soluble fraction afforded $3.37 \mathrm{~g}(63 \%)$ of a colorless liquid, $[\alpha]_{\mathrm{D}}^{23.5}=-1.26^{\circ}$ (pure, 1 $\mathrm{cm}) .[\alpha]_{\mathrm{D}}^{20.5}=-14.34^{\circ}$ (pure, $\left.\left.10 \mathrm{~cm}\right)\right]$. The NMR spectrum was identical to that for the $(S)$-(+)-isomer.

D-Cysteine methyl ester hydrochloride. D-Cys $\mathrm{HCl}_{2} \mathrm{O}$ $\left(1.02 \mathrm{~g}, 6.7 \mathrm{mmol}\right.$ ) was dehydrated at $50{ }^{\circ} \mathrm{C}, 3 \mathrm{~mm} \mathrm{Hg}$ for $2 \mathrm{~h}$ and reacted with $\mathrm{MeOH}$ saturated with anhydrous $\mathrm{HCl}$ for 20 $\mathrm{h}$ at RT. Evaporation of the solvent in vacuum resulted in $1.14 \mathrm{~g}$ (quantitative yield) of a crystalline solid with $[\alpha]_{\mathrm{D}}{ }^{24}$ $+2.22(\mathrm{c} 0.095, \mathrm{MeOH})$. [Aldrich $[\alpha]_{\mathrm{D}}{ }^{20}-1.8(\mathrm{c} 10, \mathrm{MeOH})$ for $\mathrm{L}-\mathrm{CysOMe} \mathrm{HCl}]$.

$\mathrm{N}$-(4-Nitrobenzoyl)-glycine methyl ester [methyl 2-(4nitrobenzamido)acetate, L-102]. N-(4-Nitrobenzoyl)-glycine $(2.24 \mathrm{~g}, 10 \mathrm{mmol})$ in $\mathrm{MeOH}(20 \mathrm{ml})$ and TMS-Cl $(3 \mathrm{~mL}$, $23.5 \mathrm{mmol}$ ) were left overnight at RT and the precipitated yellowish solid filtered off. Yield $1.45 \mathrm{~g}(61 \%), \mathrm{R}_{\mathrm{f}} 0.25$ (hexane-EtOAc 1:1), m.p. $157-158^{\circ} \mathrm{C}$ [[22] m.p. 150-153 $\left.{ }^{\circ} \mathrm{C}\right]$. Anal. Calcd for $\mathrm{C}_{10} \mathrm{H}_{10} \mathrm{~N}_{2} \mathrm{O}_{5}: \mathrm{C}, 50.42 ; \mathrm{H}, 4.23 ; \mathrm{N}$, 11.76. Found: C, 50.35; H, 4.22; N, 11.77 .

L-Cysteine $i$-propyl ester hydrochloride. A suspension of L-Cys $(0.3 \mathrm{~g}, 2.5 \mathrm{mmol})$ in anhydrous $i-\mathrm{PrOH}(30 \mathrm{~mL})$ and TMS-Cl $(0.85 \mathrm{~g}, 92 \mathrm{mmol})$ was heated at $70^{\circ} \mathrm{C}$ for 2 days in a pressure tube. The CysOPr- $i \mathrm{HCl}(0.5 \mathrm{~g}$, quantitative yield $)$ obtained after solvent removal was used for the next step without purification. ${ }^{1} \mathrm{H}$ NMR $\left(\mathrm{D}_{2} \mathrm{O}\right): 1.17[\mathrm{dd}, 1.2,6.3 \mathrm{~Hz}$, $\left.\mathrm{OCH}\left(\mathrm{CH}_{3}\right)_{2}\right], 2.98\left(\mathrm{dd}, 15.3,4.2 \mathrm{~Hz}, \mathrm{H}_{\beta}\right), 3.06(\mathrm{dd}, 15.3$, $\left.5.4 \mathrm{~Hz}, \mathrm{H}_{\beta},\right), 4.24\left(\mathrm{~m}, \mathrm{H}_{\alpha}\right), 5.00$ [sept, OCH $\left.\left(\mathrm{CH}_{3}\right)_{2}\right] ;{ }^{13} \mathrm{C}$ NMR $\left(\mathrm{D}_{2} \mathrm{O}\right): 21.39,24.31,55.15,73.46,168.29$.

L-S-(trans, trans-farnesyl)cysteine methyl ester. Farnesyl bromide (4.56 g, $16 \mathrm{mmol}$ ) was added to a solution of Lcysteine methyl ester hydrochloride $(2.75 \mathrm{~g}, 16 \mathrm{mmol})$ in anhydrous methanol $(28 \mathrm{~mL})$ and $7 \mathrm{~N}$ anhydrous $\mathrm{NH}_{3}$ solution in methanol $(92 \mathrm{ml})$ at $-5^{\circ} \mathrm{C}$ and the mixture stirred at this temperature for $2 \mathrm{~h}$ [23]. The solvent was evaporated under vacuum and the residue purified by flash chromatography, eluting with hexane-EtOAc 1:1 $(1.69 \mathrm{~g}, 31 \%)$. $\mathrm{R}_{\mathrm{f}} 0.2$ (hexane-EtOAc $1: 1),{ }^{1} \mathrm{H}-\mathrm{NMR}: \quad \delta 1.48 \quad\left(\mathrm{~s}, \mathrm{CH}_{3}\right), \quad 1.51$ $\left(\mathrm{s}, \mathrm{CH}_{3}\right), 1.59\left(\mathrm{~s}, \mathrm{CH}_{3}\right), 1.66\left(\mathrm{~s}, \mathrm{CH}_{3}\right), 1.59\left(\mathrm{~s}, \mathrm{CH}_{3}\right), 1.65$ (s, $\mathrm{NH}_{2}$ ), 1.80-2.10 (m, $4 \mathrm{CH}_{2}$ ), 2.61 and 2.77 (dd, S-C-H $J=7.5 \mathrm{~Hz}$ ), 3.09 (d, $\left.\mathrm{CH}_{2} \mathrm{~S}, J=7.2 \mathrm{~Hz}\right), 3.55(\mathrm{dd}, \alpha-\mathrm{H}, J=3.0 \mathrm{~Hz}$ ), $3.65\left(\mathrm{~s}, \mathrm{OCH}_{3}\right), 5.04$ (broad, $\left.2 \times \mathrm{C}=\mathrm{CH}\right), 5.14(\mathrm{t}, \mathrm{C}=\mathrm{C} \underline{\mathrm{HCS}}$, $J=1.2 \mathrm{~Hz}) ;{ }^{13} \mathrm{C}-\mathrm{NMR}: \delta 16.17,16.26,17.84,25.85,25.48$, $26.55,29.94,36.48,39.77,39.85,52.27,54.39,120.2,123.9$ 124.5, 131.3, 135.4, 139.6, 174.6.

D-S-(trans, trans-farnesyl)cysteine methyl ester. Farnesyl chloride $(626 \mathrm{mg}, 2.7 \mathrm{mmol})$ was added to a solution of D-CysOMe $\mathrm{HCl}(446 \mathrm{mg}, 2.6 \mathrm{mmol}$ ) in anhydrous methanol $(1 \mathrm{ml})$ and $7 \mathrm{~N}$ anhydrous $\mathrm{NH}_{3}$ solution in methanol $(2 \mathrm{~mL})$ at $-5^{\circ} \mathrm{C}$. The reaction mixture was stirred at $-5^{\circ} \mathrm{C}$ for $10 \mathrm{~min}$, at $0^{\circ} \mathrm{C}$ for $30 \mathrm{~min}$ and at RT for $45 \mathrm{~min}$. After an identical treatment, the product $(635 \mathrm{mg}, 72 \%)$ with $[\alpha]_{\mathrm{D}}{ }^{20}-6.35$ (c $0.1, \mathrm{CHCl}_{3}$ ), $[\alpha]_{\mathrm{D}}^{21}-12.3$ (c $0.1, \mathrm{PhMe}$ ) displayed the same NMR spectra and $\mathrm{R}_{\mathrm{f}}$ as the $\mathrm{L}$-isomer.

L-S-(trans-geranyl)cysteine methyl ester. Geranyl bromide $(1.3 \mathrm{~mL}, 6.6 \mathrm{mmol})$ was added to a stirred solution of L-cysteine methyl ester hydrochloride $(1.02 \mathrm{~g}, 6 \mathrm{mmol})$ in anhydrous methanol $(8 \mathrm{ml})$ and $7 \mathrm{~N}$ anhydrous $\mathrm{NH}_{3}$ solution in anhydrous methanol $(15 \mathrm{~mL})$ at $-10^{\circ} \mathrm{C}$. The mixture was kept at $-10^{\circ} \mathrm{C}$ for $10 \mathrm{~min}$, at $0^{\circ} \mathrm{C}$ for $50 \mathrm{~min}$ and at $\mathrm{RT}$ for $1 \mathrm{~h}$. The solvent was removed under vacuum and the residue suspended in EtOAc. The solid was removed by centrifugation and the organic phase evaporated. The product was applied onto a column and eluted with a gradient of DCM DCM-EtOAc 30:1 (699 mg, 43\%). $\mathrm{R}_{\mathrm{f}} 0.2$ (hexane-EtOAc $1: 2),[\alpha]_{\mathrm{D}}{ }^{22}+17.0\left(\mathrm{c} 0.22\right.$, PhMe). ${ }^{1} \mathrm{H}-\mathrm{NMR}: \delta 1.53\left(\mathrm{~s}, \mathrm{CH}_{3}\right)$, $1.59\left(\mathrm{~s}, \mathrm{CH}_{3}\right), 1.61\left(\mathrm{~s}, \mathrm{CH}_{3}\right), 1.65\left(\mathrm{~s}, \mathrm{NH}_{2}\right), 1.80-2.20(\mathrm{~m}, 4$ $\left.\mathrm{CH}_{2}\right), 2.61\left(\mathrm{dd}, 13.5,7.5 \mathrm{~Hz}, \mathrm{H}_{\beta}\right), 2.80(\mathrm{dd}, 13.5,4.8 \mathrm{~Hz}$, $\left.\mathrm{H}_{\beta}\right), 3.11\left(\mathrm{~m}, \mathrm{CH}_{2} \mathrm{~S}\right), 3.55\left(\mathrm{dd}, 4.8,7.5 \mathrm{~Hz}, \mathrm{H}_{\alpha}\right), 3.66$ $\left(\mathrm{s}, \mathrm{OCH}_{3}\right), 5.02(\mathrm{~m}, \mathrm{C}=\mathrm{CH}), 5.17(\mathrm{~m}, \mathrm{C}=\mathrm{CHCS}) ;{ }^{13} \mathrm{C}-\mathrm{NMR}$ : $\delta 16.2,17.7,25.6,26.7,30.2,36.8,39.7,52.0,54.7,120.4$, 124.1, 131.6, 139.4, 174.5.

D-N-Acetyl-S-(trans, trans-farnesyl)cysteine methyl ester [(S)-methyl 3-((2E,6E)-3,7,11-trimethyldodeca-2,6,10trienylthio)-2-acetamidopropanoate, L-69]. D-(S-trans, transfarnesyl)cysteine methyl ester (106 $\mathrm{mg}, 0.3 \mathrm{mmol})$ was reacted with acetic anhydride $(77 \mathrm{mg}, 0.75 \mathrm{mmol})$ and $\mathrm{K}_{2} \mathrm{CO}_{3}(189 \mathrm{mg}, 1.36 \mathrm{mmol})$ in PhMe-water $(1.5+1 \mathrm{~mL})$. The organic phase was dried over $\mathrm{K}_{2} \mathrm{CO}_{3}$, the solvent removed and the residue purified by column chromatography eluting with hexane-EtOAc 1:1.5 to afford the product (114 $\mathrm{mg}, 96 \%$ ). $\mathrm{R}_{\mathrm{f}} 0.3$ (hexane-EtOAc 1:1), $[\alpha]_{\mathrm{D}}{ }^{22}+25.4$ (c 0.025 , acetone). ${ }^{1} \mathrm{H}$-NMR: $\delta 1.57\left(\mathrm{~s}, \mathrm{CH}_{3}\right), 1.64\left(\mathrm{~s}, \mathrm{CH}_{3}\right), 1.64$ (s, $\left.\mathrm{CH}_{3}\right), 1.65\left(\mathrm{~d}, 0.9 \mathrm{~Hz}, \mathrm{CH}_{3}\right), 1.87-2.10\left(\mathrm{~m}, 8 \mathrm{CH}_{2}\right), 2.02$ $\left(\mathrm{s}, \mathrm{COCH}_{3}\right), 2.84\left(\mathrm{dd}, 13.8,5.7 \mathrm{~Hz}, \mathrm{H}_{\beta}\right), 2.93(\mathrm{dd}, 13.8,5.1$ $\left.\mathrm{Hz}, \mathrm{H}_{\beta}\right), 3.13\left(\mathrm{~m}, \mathrm{CH}_{2} \mathrm{~S}\right), 3.73\left(\mathrm{~s}, \mathrm{OCH}_{3}\right), 4.78\left(\mathrm{~m}, \mathrm{H}_{\alpha}\right), 5.06$ $(\mathrm{m}, 2 \mathrm{C}=\mathrm{CH}), 5.17(\mathrm{~m}, \mathrm{C}=\mathrm{CHCS}), 6.31(\mathrm{~d}, \mathrm{CONH}) ;{ }^{13} \mathrm{C}-$ NMR: $\delta$ 16.2, 16.3, 17.9, 23.3, 25.9, 26.6, 26.9, 30.2, 33.6, $39.8,39.9,52.0,52.7,119.8,123.9,124.5,131.5,135.6$, 140.2, 170.0, 171.7. Anal. Calcd for $\mathrm{C}_{21} \mathrm{H}_{35} \mathrm{NO}_{3} \mathrm{~S}$ S.0.6 $\mathrm{H}_{2} \mathrm{O}: \mathrm{C}$, 64.28; H, 9.30; N, 3.57. Found: C, 64.02; H, 9.03; N, 3.52.

L-N-(4-Nitrobenzoyl)-S-(trans, trans-farnesyl)cysteine methyl ester $[(R)$-methyl 3-((2E,6E)-3,7,11-trimethyldodeca2,6,10-trienylthio)-2-(4-nitrobenzamido)propanoate, RDPNB]. Triethylamine $(181 \mu \mathrm{l}, 1.32 \mathrm{mmol})$ was added to a stirred suspension of $p$-nitrobenzoyl chloride $(245 \mathrm{mg}, 1.32$ mmol) and L-S-(trans,trans-farnesyl)cysteine methyl ester $(412 \mathrm{mg}, 1.2 \mathrm{mmol})$ in anhydrous DCM $(10 \mathrm{ml})$ on ice. After 10 min the mixture was left at RT for $4 \mathrm{~h}$. The solvent was removed and the solid residue was dissolved in ethyl acetate and washed with $5 \% \mathrm{NaHCO}_{3}, 10 \% \mathrm{HCl}$ and brine. It was then dried over $\mathrm{MgSO}_{4}$ and the solvent evaporated. The resulting oil was purified by column chromatography eluting with DCM to give a yellowish solid (180 mg, 30\%) with a m.p. of $52-54.5{ }^{\circ} \mathrm{C} ;[\alpha]_{\mathrm{D}}{ }^{20}=-66.0^{\circ}(\mathrm{c}=0.015$, acetone). IR (KBr) $\mathrm{NH}$ (3300), $\mathrm{C}=\mathrm{O}$ (1750), $\mathrm{O}=\mathrm{CN}$ (1620), $\mathrm{NO}_{2}$ (1355), and $\mathrm{OMe}(1250) \mathrm{cm}^{-1} ; \mathrm{R}_{\mathrm{f}}$ (hexane-EtOAc 1:1) 0.65 amide, 0.25 amine. ${ }^{1} \mathrm{H}-\mathrm{NMR}: \delta 1.58\left(\mathrm{~s}, \mathrm{CH}_{3}\right), 1.42\left(\mathrm{~s}, \mathrm{CH}_{3}\right), 1.66$ (s, $\left.\mathrm{CH}_{3}\right), 1.95-2.10\left(\mathrm{~m}, 4 \times \mathrm{CH}_{2}\right), 3.00-3.07(\beta$ ' and $\beta$ ) (dd, S-C-H $\quad J=7.5 \mathrm{~Hz}), \quad 3.09-3.21 \quad\left(\mathrm{t}, \quad \mathrm{CH}_{2} \mathrm{~S}, \quad J=7.2 \mathrm{~Hz}\right), \quad 3.81$ $\left(\mathrm{s}, \mathrm{OCH}_{3}\right), 4.97-5.04$ (d, $\left.\underline{\mathrm{HC}-\mathrm{NH}_{2}}\right)$, 5.04-5.06 (broad, 2 
$\mathrm{C}=\mathrm{C} \underline{\mathrm{H}}$ ), 5.09-5.15 (broad, $\mathrm{C}=\mathrm{CHCS}, J=1.2 \mathrm{H}), 7.12(\mathrm{~d}, \mathrm{HN}$, $J=10.2 \mathrm{~Hz}$ ), 7.98 (m, Ar, 2H), 8.28 (m, Ar, 2H). ${ }^{13} \mathrm{C}-\mathrm{NMR}: \delta$ 16.27, 16.39, 17.95, 25.97, 26.64, 26.93, 30.07, 33.28, 39.87, 39.27, 43.71, 52.25, 53.08, 119.6, 123.8, 124.0, 124.5, 128.7, 131.6, 135.7, 139.2, 140.5, 149.9, 165.8, 168.9, 171.3. Anal. Calcd for $\mathrm{C}_{26} \mathrm{H}_{36} \mathrm{~N}_{2} \mathrm{O}_{5} \mathrm{~S}$ : C, 63.91; H, 7.43; N, 5.73. Found: C, 63.92; H, 7.44; N, 5.67.

D-N-(4-Nitrobenzoyl)-S-(trans, trans-farnesyl)cysteine methyl ester $[(S)$-methyl 3-((2E,6E)-3,7,11-trimethyldodeca2,6,10-trienylthio)-2-(4-nitrobenzamido)propanoate, L-70]. Triethylamine $(0.2 \mathrm{~mL}, 1.4 \mathrm{mmol})$ was added to a stirred suspension of $p$-nitrobenzoyl chloride $(238 \mathrm{mg}, 1.28 \mathrm{mmol}$ ) and D-S-(trans,trans-farnesyl)-cysteine methyl ester (396 $\mathrm{mg}, 1.17 \mathrm{mmol})$ in dry $\mathrm{CHCl}_{3}(3 \mathrm{ml})$ at $-10^{\circ} \mathrm{C}$. The mixture was left in a fridge overnight and treated as described for the L-isomer, giving a quantitative yield of a yellowish solid with m.p. $57-58^{\circ} \mathrm{C} ;[\alpha]_{\mathrm{D}}{ }^{22}=+79.3^{\circ}(\mathrm{c}=0.015$, acetone $)$ and the same NMR spectrum and $\mathrm{R}_{\mathrm{f}}$ as the L-isomer. Anal. Calcd for $\mathrm{C}_{26} \mathrm{H}_{36} \mathrm{~N}_{2} \mathrm{O}_{5} \mathrm{~S}$ : C, 63.91; H, 7.43; N, 5.73. Found: C, 63.99; H, 7.43; N, 5.71 .

$\mathrm{N}-(4-N i t r o b e n z o y l)-S-($ trans, trans, trans-geranylgeranyl) cysteine methyl ester $[(R)$-methyl 3-((2E,6E,10E)-3,7,11,15tetramethylhexadeca-2,6,10,14-tetraenylthio)-2-(4-nitrobenzamido)propanoate, L-80]. trans, trans, trans-Geranylgeranyl bromide $(1.29 \mathrm{~g}, 3.7 \mathrm{mmol})$ dissolved in anhydrous ether $(3 \mathrm{ml})$ was added to a solution of L-cysteine methyl ester $(0.68 \mathrm{~g}, 3.7 \mathrm{mmol})$ in methanol $(2 \mathrm{ml})$ and $7 \mathrm{~N}$ solution of $\mathrm{NH}_{3}$ in methanol $(3.5 \mathrm{ml}, 24.5 \mathrm{mmol})$ at -5 to $-10^{\circ} \mathrm{C}$. The reaction mixture was stirred at $-5^{\circ} \mathrm{C}$ for $10 \mathrm{~min}$, at $0^{\circ} \mathrm{C}$ for $30 \mathrm{~min}$ and at RT $45 \mathrm{~min}$. The solvent was evaporated under vacuum and the residue taken into EtOAc. The organic phase was rotovaped and the product purified on a column eluting with hexane-EtOAc $1: 1 \quad(1.14 \mathrm{~g}, 75 \%) . \quad \mathrm{R}_{\mathrm{f}} 0.15$ (hexane-EtOAc 1:1). A solution of L-S-(trans, trans, transgeranylgeranyl)cysteine methyl ester $(1.14 \mathrm{~g}, 2.8 \mathrm{mmol})$ in EtOAc $(50 \mathrm{~mL})$ was stirred with $p$-nitrobenzoyl chloride $(0.64 \mathrm{~g}, 3.4 \mathrm{mmol})$ and a solution of $\mathrm{K}_{2} \mathrm{CO}_{3}(0.77 \mathrm{~g}, 5.6$ $\mathrm{mmol})$ in water $(5 \mathrm{~mL})$. After completion of the reaction (TLC, $\mathrm{R}_{\mathrm{f}}$ hexane-EtOAc 1:1 0.2 amine, 0.8 amide), the organic phase was separated, washed with diluted $\mathrm{NaOH}$, brine, dried over $\mathrm{K}_{2} \mathrm{CO}_{3}$ and the solvent removed under vacuum. Column chromatography (gradient from DCM to DCM-EtOAc 30:1) afforded $1.12 \mathrm{~g}(72 \%)$ of a light yellow solid, $\mathrm{R}_{\mathrm{f}}$ (hexane-EtOAc $\left.1: 1\right)$ m.p. $45-46^{\circ} \mathrm{C},[\alpha]_{\mathrm{D}}{ }^{23}-39.4^{\circ}$ (c 0.08, acetone). ${ }^{1} \mathrm{H}-\mathrm{NMR}: \delta 1.57\left(\mathrm{~s}, 3 \mathrm{CH}_{3}\right), 1.63\left(\mathrm{~s}, \mathrm{CH}_{3}\right)$, $1.65\left(\mathrm{~s}, \mathrm{CH}_{3}\right), 1.90-2.05\left(\mathrm{~m}, 6 \mathrm{CH}_{2}\right), 2.95-3.15\left(\mathrm{~m}, \mathrm{H}_{\beta, \beta},+\right.$ $\left.\mathrm{CH}_{2} \mathrm{~S}\right), 3.79\left(\mathrm{~s}, \mathrm{OCH}_{3}\right), 4.96\left(\mathrm{~m}, \mathrm{H}_{\mathrm{a}}, 1 \mathrm{H}\right), 5.06(\mathrm{~m}, 3 \mathrm{C}=\mathrm{C} \underline{\mathrm{H}})$, $5.18(\mathrm{~m}, \mathrm{C}=\mathrm{CHCS}, 1 \mathrm{H}), 7.10(\mathrm{~d}, \mathrm{HN}, J=6.6 \mathrm{~Hz}), 7.96$ (m, Ar, 2H), 8.25 (m, Ar, 2H). ${ }^{13} \mathrm{C}-\mathrm{NMR}: \delta$ 16.21, 16.24, 16.37, 17.95, 25.97, 26.63, 26.83, 26.97, 30.19, 33.45, 39.84, 39.89, 39.92, 52.53, 53.06, 119.55, 123.85, 124.04, 124.36, $124.59,128.62,131.42,135.17,135.67,139.46,140.54$, 150.03, 165.27, 171.3. Anal. Calcd for $\mathrm{C}_{31} \mathrm{H}_{44} \mathrm{~N}_{2} \mathrm{O}_{5} \mathrm{~S}$ : C, 66.88; H, 7.97; N, 5.03. Found: C, 66.85; H, 7.95; N, 5.07.

L-N-(4-Nitrobenzoyl)-S-(trans-geranyl)cysteine methyl ester [(R)-methyl 3-((E)-3,7-dimethylocta-2,6-dienylthio)-2(4-nitrobenzamido)propanoate, L-72]. This was synthesized from L-(S-trans-geranyl)cysteine methyl ester $(699 \mathrm{mg}, 2.6$ mmol), p-nitrobenzoyl chloride (530 $\mathrm{mg}, 2.9 \mathrm{mmol})$ and $\mathrm{K}_{2} \mathrm{CO}_{3}$ (790 mg, $5.7 \mathrm{mmol}$ ) in water - EtOAc. A light yellow solid was obtained, m.p. $60-65^{\circ} \mathrm{C},[\alpha]_{\mathrm{D}}^{25}-78.0^{\circ}$ (c 0.1 , acetone). ${ }^{1} \mathrm{H}-\mathrm{NMR}: \delta 1.55\left(\mathrm{~s}, \mathrm{CH}_{3}\right), 1.61\left(\mathrm{~s}, \mathrm{CH}_{3}\right), 1.63$ $\left(\mathrm{s}, \mathrm{CH}_{3}\right), 1.90-2.10\left(\mathrm{~m}, 2 \mathrm{CH}_{2}\right), 2.29-3.22\left(\mathrm{~m}, \mathrm{H}_{\beta, \beta},+\mathrm{CH}_{2} \mathrm{~S}\right)$, $3.77\left(\mathrm{~s}, \mathrm{OCH}_{3}\right), 4.94\left(\mathrm{~m}, \mathrm{H}_{\alpha}\right), 5.12(\mathrm{~m}, \mathrm{C}=\mathrm{CH}), 5.15$ $(\mathrm{m}, \mathrm{C}=\mathrm{CHCS}), 7.15(\mathrm{~d}, \mathrm{HN}), 7.96(\mathrm{~m}, \mathrm{Ar}, 2 \mathrm{H}), 8.23(\mathrm{~m}, \mathrm{Ar}$, 2H). ${ }^{13}$ C-NMR: $\delta 16.3,17.9,25.9,26.6,30.1,33.3,39.8$, 52.5, 53.1, 119.6, 123.9, 124.0, 128.6, 132.0, 139.4, 140.4, 150.0, 165.3, 171.4. Anal. Calcd for $\mathrm{C}_{21} \mathrm{H}_{28} \mathrm{~N}_{2} \mathrm{O}_{5} \mathrm{~S}$ : C, 59.98; H, 6.71; N, 6.66. Found: C, 89.80; H, 6.66; N, 6.60.

L-N-(4-Nitrobenzoyl)-[S-(S)-citronellyl]cysteine methyl ester $[(R)$-methyl 3-((S)-3,7-dimethyloct-6-enylthio)-2-(4nitrobenzamido)propanoate, L-83]. (S)-(+)-Citronellyl iodide (1.33 g, $5 \mathrm{mmol})$ was added to a stirred solution of Lcysteine methyl ester hydrochloride $(0.858 \mathrm{~g}, 5 \mathrm{mmol})$ in anhydrous methanol $(2 \mathrm{~mL})$ and $7 \mathrm{~N}$ anhydrous $\mathrm{NH}_{3}$ solution in methanol $(4.8 \mathrm{~mL})$ at $-10^{\circ} \mathrm{C}$. The mixture was kept for 10 min at $-10^{\circ} \mathrm{C}$ followed by continuous stirring without cooling for $1 \mathrm{~h}$. The solvent was evaporated in vacuum, the residue suspended in EtOAc, the solid filtered off and the organic phase was evaporated. The resulting residue was applied onto a column and eluted with hexane-EtOAc 1:2 followed by EtOAc. Fractions containing L-S- $(S)$ citronellyl)cysteine methyl ester $\left(\mathrm{R}_{\mathrm{f}} 0.25\right.$ hexane-EtOAc $\left.1: 2\right)$ were combined, shaken with $p$-nitrobenzoyl chloride $(1.04 \mathrm{~g}$, $5.4 \mathrm{mmol})$ and a solution of $\mathrm{K}_{2} \mathrm{CO}_{3}(1.38 \mathrm{~g}, 10 \mathrm{mmol})$ in water $(10 \mathrm{~mL})$ and treated as above. Column chromatography (gradient from DCM to DCM-EtOAc 50:1) afforded $0.979 \mathrm{~g}(46 \%$ yield $)$ of a light yellow solid, $\mathrm{R}_{\mathrm{f}} 0.6$, hexaneEtOAc $1: 1$, m.p. $47.5-48.5^{\circ} \mathrm{C},[\alpha]_{\mathrm{D}}{ }^{25}-45.8^{\circ}$ (c 0.08 , acetone). ${ }^{1} \mathrm{H}-\mathrm{NMR}: \delta 0.84\left(\mathrm{~d}, 6.3 \mathrm{~Hz}, \mathrm{CHCH}_{3}\right), 1.12\left(\mathrm{~m}, \mathrm{H}_{4}, 1 \mathrm{H}\right)$, 1.20-1.60 (m, $\left.\mathrm{H}_{4},+\mathrm{H}_{5,5}, \mathrm{CHCH}_{3}, 4 \mathrm{H}\right), 1.56\left(\mathrm{~s}, \mathrm{CH}_{3}\right), 1.65$ (s, $\left.\mathrm{CH}_{3}\right), 1.92\left(\mathrm{~m}, \mathrm{H}_{5,5},\right), 2.29\left(\mathrm{~m}, \mathrm{CH}_{2} \mathrm{~S}\right), 3.11\left(\mathrm{~m}, \mathrm{H}_{\beta}, \beta\right), 3.80$ $\left(\mathrm{s}, \mathrm{OCH}_{3}\right), 5.00\left(\mathrm{~m}, \mathrm{H}_{\alpha}+\mathrm{C}=\mathrm{CH}\right), 7.11(\mathrm{~d}, \mathrm{HN}), 7.97(\mathrm{~m}, \mathrm{Ar}$, 2H), 8.28 (m, Ar, 2H). ${ }^{13} \mathrm{C}-\mathrm{NMR}: \delta 17.85,19.37,25.59$, $25.89,30.79,32.03,34.32,36.89,36.92,52.60,53.09$, 124.07, 124.67, 128.62, 131.59, 139.44, 150.06, 165.21, 171.30. Anal. Calcd for $\mathrm{C}_{21} \mathrm{H}_{30} \mathrm{~N}_{2} \mathrm{O}_{5} \mathrm{~S}: \mathrm{C}, 59.69 ; \mathrm{H}, 7.16 ; \mathrm{N}$, 6.63. Found: C, 59.94; H, 7.05; N, 6.55.

L-N-(4-Nitrobenzoyl)-[S- $(R)$-citronellyl]cysteine methyl ester $[(R)$-methyl 3- $((R)-3,7-$ dimethyloct-6-enylthio)-2-(4nitrobenzamido)propanoate, L-86]. $(R)$-(-)-citronellyl iodide was used for the synthesis in essentially the same procedure as for L-N-(4-Nitrobenzoyl)-[S- $(S)$-citronellyl]cysteine methyl ester. Yield $1.478 \mathrm{~g}(70 \%)$, m.p. $57^{\circ} \mathrm{C},[\alpha]_{\mathrm{D}}{ }^{23}-57.4^{\circ}$ (c 0.09, acetone). ${ }^{1} \mathrm{H}-\mathrm{NMR}: \delta 0.81\left(\mathrm{~d}, 6.3 \mathrm{~Hz}, \mathrm{CHCH}_{3}\right)$, 1.00-1.60 (m, H $\left.{ }_{4,4}+\mathrm{H}_{5,5},+\mathrm{CHCH}_{3}, 5 \mathrm{H}\right), 1.54\left(\mathrm{~s}, \mathrm{CH}_{3}\right), 1.62$ (s, $\left.\mathrm{CH}_{3}\right), 1.90-2.10\left(\mathrm{~m}, \mathrm{H}_{5,5},\right), 2.49\left(\mathrm{~m}, \mathrm{CH}_{2} \mathrm{~S}\right), 3.04$ (dd, 5.4, $\left.13.8 \mathrm{~Hz}, \mathrm{H}_{\beta}\right), 3.12\left(\mathrm{dd}, 4.8,13.8 \mathrm{~Hz}, \mathrm{H}_{\beta}\right), 3.77\left(\mathrm{~s}, \mathrm{OCH}_{3}\right)$, $4.95\left(\mathrm{~m}, \mathrm{H}_{\alpha}\right), 5.00(\mathrm{~m}, \mathrm{C}=\mathrm{CH}), 7.19(\mathrm{~d}, \mathrm{HN}), 7.95(\mathrm{~m}, \mathrm{Ar}$, 2H), $8.24(\mathrm{~m}, \mathrm{Ar}, 2 \mathrm{H}) .{ }^{13} \mathrm{C}-\mathrm{NMR}: \delta 17.83,19.34,25.59$, $25.87,30.71,32.01,34.25,36.83,36.91,52.61,53.06$, $124.01,124.69,128.64,131.53,139.42,150.01,165.28$, 171.33. Anal. Calcd for $\mathrm{C}_{21} \mathrm{H}_{30} \mathrm{~N}_{2} \mathrm{O}_{5} \mathrm{~S}: \mathrm{C}, 59.69 ; \mathrm{H}, 7.16 ; \mathrm{N}$, 6.63. Found: C, 59.93; H, 7.12; N, 6.55.

L-N-(4-Nitrobenzoyl)-S-(ethyl)cysteine methyl ester [(R)-methyl 2-(4-nitrobenzamido)-3-(ethylthio)propanoate, L-74]. L-(S-ethyl)cysteine (1.49 g, $10 \mathrm{mmol})$ was reacted overnight with TMS-Cl (3 ml, $23.5 \mathrm{mmol})$ in $\mathrm{MeOH}$ (13 $\mathrm{mL})$ at RT, giving L-(S-ethyl)cysteine methyl ester. After removal of solvent, the solid was reacted with $p$-nitrobenzoyl chloride $(2.33 \mathrm{~g}, 12 \mathrm{mmol})$ and $\mathrm{K}_{2} \mathrm{CO}_{3}(2.76 \mathrm{~g}, 20 \mathrm{mmol})$ in 
water - EtOAc $(10+20 \mathrm{ml})$ and treated as above. Column chromatography (gradient from DCM to DCM-EtOAc 40:1) afforded $1.36 \mathrm{~g}(43 \%)$ of a yellowish solid, m.p. $95-96^{\circ} \mathrm{C}$, $[\alpha]_{\mathrm{D}}{ }^{21}-56.1^{\mathrm{o}}$ (c 0.065 , acetone). $\mathrm{R}_{\mathrm{f}} 0.45$ (hexane-EtOAc $1: 1$ ). ${ }^{1} \mathrm{H}-\mathrm{NMR}: \delta 1.22\left(\mathrm{t}, \mathrm{CH}_{3}\right), 2.53\left(\mathrm{q}, \mathrm{CH}_{2}\right), 3.22\left(\mathrm{~m}, \mathrm{H}_{\beta}, \beta\right)$, $3.80\left(\mathrm{~s}, \mathrm{OCH}_{3}\right), 4.98\left(\mathrm{~m}, \mathrm{H}_{\alpha}\right), 7.11(\mathrm{~d}, \mathrm{HN}), 7.97(\mathrm{~m}, \mathrm{Ar}$, 2H), 8.28 (m, Ar, 2H). ${ }^{13} \mathrm{C}-\mathrm{NMR}: \delta 14.9,26.8,33.9,52.5$, 53.1, 124.1, 128.6, 139.4, 150.0, 165.2, 171.3. Anal. Calcd for $\mathrm{C}_{13} \mathrm{H}_{16} \mathrm{~N}_{2} \mathrm{O}_{5} \mathrm{~S}$ : C, 49.99; H, 5.16; N, 8.97. Found: C, $50.18 ; \mathrm{H}, 5.04 ; \mathrm{N}, 8.81$.

L-N-(4-Nitrobenzoyl)-S-( $i$-amyl)cysteine methyl ester $[(R)$-methyl 2-(4-nitrobenzamido)-3-(isopentylthio)propanoate, L-81]. $i$-Amyl bromide $(0.615 \mathrm{~g}, 4.1 \mathrm{mmol})$ was added to a solution of L-cysteine methyl ester $(0.68 \mathrm{~g}, 3.7 \mathrm{mmol})$ in methanol $(2 \mathrm{~mL})$ and $7 \mathrm{~N}$ solution of $\mathrm{NH}_{3}$ in methanol (3.5 mL, $24.5 \mathrm{mmol})$ at $-5--10^{\circ} \mathrm{C}$. The reaction mixture was stirred for $30 \mathrm{~min}$ at $-5^{\circ} \mathrm{C}, 1 \mathrm{~h}$ at $0^{\circ} \mathrm{C}$ and $1 \mathrm{~h}$ at RT. The solvent was evaporated in vacuum, the residue taken into EtOAc, the organic phase rotovaped and the residue purified on a column in EtOAc $(1.14 \mathrm{~g}, 75 \%)$. Fractions containing $\mathrm{L}-\left(\mathrm{S}-i\right.$-amyl)cysteine methyl ester $\left(\mathrm{R}_{\mathrm{f}} 0.15\right.$, hexane-EtOAc 1:1) were combined and shaken with $p$-nitrobenzoyl chloride $(0.64 \mathrm{~g}, 3.4 \mathrm{mmol})$ and a solution of $\mathrm{K}_{2} \mathrm{CO}_{3}(0.77 \mathrm{~g}, 5.6$ $\mathrm{mmol})$ in water $(5 \mathrm{~mL})$ in a separatory funnel for $5 \mathrm{~min}$. After completion of the reaction (TLC in EtOAc 1:1, $\mathrm{R}_{\mathrm{f}}$ of amide 0.65 ) the organic phase was separated, washed with diluted $\mathrm{NaOH}$, brine, dried over $\mathrm{K}_{2} \mathrm{CO}_{3}$ and the solvent removed in vacuum. Column chromatography (gradient from DCM to DCM-EtOAc 20:1) afforded $0.49 \mathrm{~g} \mathrm{(38 \% )}$ of a light yellow solid, m.p. $93-93.5^{\circ} \mathrm{C},[\alpha]_{\mathrm{D}}{ }^{25}-53.3^{\circ}$ (c 0.05 , acetone). ${ }^{1} \mathrm{H}-\mathrm{NMR}: \delta 0.85\left(\mathrm{dd}, 6.6,1.5 \mathrm{~Hz}, \mathrm{CHCH}_{3}\right), 1.42(\mathrm{~m}$, $\left.\mathrm{CH}_{2} \mathrm{CH}_{2} \mathrm{~S}, 2 \mathrm{H}\right), 1.67$ (m, $\underline{\mathrm{HMe}}_{2}$ ), $2.51\left(\mathrm{~m}, \overline{\mathrm{CH}}_{2} \mathrm{CH}_{2} \mathrm{~S}\right), 3.11$ $\left(\mathrm{H}_{\beta}, \beta, \mathrm{m},\right), 3.80\left(\mathrm{~s}, \mathrm{OCH}_{3}\right), 4.98\left(\mathrm{H}_{\mathrm{a}}, \mathrm{m}\right), 7.11$ (d, HN, broad), 7.97 (m, Ar, 2H), 8.28 (m, Ar, 2H). ${ }^{13} \mathrm{C}-\mathrm{NMR}: \delta$ 22.40, 27.55, 30.98, 34.29, 38.68, 52.59, 53.10, 124.07, 128.62, 139.45, 150.06, 165.23, 171.31. Anal. Calcd for $\mathrm{C}_{16} \mathrm{H}_{22} \mathrm{~N}_{2} \mathrm{O}_{5} \mathrm{~S}$ : C, 54.22; H, 6.26; N, 7.90. Found: C, 54.23; $\mathrm{H}, 6.30 ; \mathrm{N}, 7.85$.

L-N-(4-Nitrobenzoyl)-S-(prenyl)cysteine methyl ester [(R)-methyl 3-(3-methylbut-2-enylthio)-2-(4-nitrobenzamido) propanoate, L-77]. A solution of $7 \mathrm{~N}$ ammonia in $\mathrm{MeOH}(70$ mmol, $10 \mathrm{~mL}$ ) was added to a solution of L-CysOMe $\mathrm{HCl}$ $(1.03 \mathrm{~g}, 6 \mathrm{mmol})$ in anhydrous $\mathrm{MeOH}(2 \mathrm{~mL})$ that had been cooled to $-5^{\circ} \mathrm{C}$. Prenyl chloride $(0.69 \mathrm{~g}, 6.6 \mathrm{mmol})$ was then added in one portion. After $15 \mathrm{~min}$ at $-5^{\circ} \mathrm{C}$, the mixture was put on ice for $30 \mathrm{~min}$, the solvent removed in vacuum, the residue taken into EtOAc, precipitated ammonium chloride extracted with water and the organic phase vigorously shaken with $p$-nitrobenzoyl chloride $(1.22 \mathrm{~g}, 6.6 \mathrm{mmol})$ and a solution of $\mathrm{K}_{2} \mathrm{CO}_{3}(1.66 \mathrm{~g}, 12 \mathrm{mmol})$ in water $(7.7 \mathrm{~mL})$. After completion of the reaction, the organic phase was separated, dried over $\mathrm{K}_{2} \mathrm{CO}_{3}$ and the solvent evaporated in vacuum. Column chromatography (gradient from DCM to DCM-EtOAc 10:7) afforded $1.44 \mathrm{~g}$ (68\% for 2 steps) of a yellowish solid with m.p. $89-90^{\circ} \mathrm{C},[\alpha]_{\mathrm{D}}{ }^{24}-57.4^{\circ}$ (c 0.07 , acetone), $\mathrm{R}_{\mathrm{f}} 0.7$ (hexane-EtOAc 1:2). ${ }^{1} \mathrm{H}-\mathrm{NMR}: \quad \delta 1.63$ $\left(\mathrm{s}, \mathrm{CH}_{3}\right), 1.70\left(\mathrm{~s}, \mathrm{CH}_{3}\right), 2.95-3.15\left(\mathrm{~m}, \mathrm{H}_{\beta, \beta},+\mathrm{CH}_{2} \mathrm{~S}\right) 3.81$ $\left(\mathrm{s}, \mathrm{OCH}_{3}\right), 4.97\left(\mathrm{~m}, \mathrm{H}_{\alpha}\right), 5.17(\mathrm{~m}, \mathrm{C}=\mathrm{CH}), 7.06(\mathrm{~d}, \mathrm{NH}), 7.98$ $(\mathrm{m}, \mathrm{Ar}, 2 \mathrm{H}), 8.28(\mathrm{~m}, \mathrm{Ar}, 2 \mathrm{H})$; ${ }^{13} \mathrm{C}-\mathrm{NMR}: \delta \mathrm{18.0}, 25.9$, 26.55, 30.4, 33.6, 52.5, 53.1, 119.8, 124.1, 128.6,136.9, 139.5, 165.2, 171.3. Anal. Calcd for $\mathrm{C}_{16} \mathrm{H}_{20} \mathrm{~N}_{2} \mathrm{O}_{5} \mathrm{~S}$ : C, 54.53; H, 5.72; N, 7.95. Found: C, 54.54; H, 5.68; N, 7.85.
L-N-(4-Nitrobenzoyl)-S-(benzyl)cysteine methyl ester [(R)-methyl 2-(4-nitrobenzamido)-3-(benzylthio)propanoate, L-93]. A suspension of L-Cys(Bn)OMe $\mathrm{HCl}(1.36 \mathrm{~g}, 5$ mmol) in EtOAc $(50 \mathrm{~mL})$ was shaken with $p$-nitrobenzoyl chloride $(1.37 \mathrm{~g}, 6 \mathrm{mmol})$ and a solution of $\mathrm{K}_{2} \mathrm{CO}_{3}(2.07 \mathrm{~g}$, $15 \mathrm{mmol})$ in water $(15 \mathrm{~mL})$. After completion of the reaction, concentrated $\mathrm{NH}_{4} \mathrm{OH}(1 \mathrm{~mL})$ was added, the mixture was kept at RT for $15 \mathrm{~min}$ and the organic phase was treated as above. Column chromatography (gradient from DCM to DCM-EtOAc 30:1) afforded $1.27 \mathrm{~g}(68 \%)$ of a yellowish solid with m.p. $114-115^{\circ} \mathrm{C},[\alpha]_{\mathrm{D}}{ }^{26}-77.4^{\circ}$ (c 0.05 , acetone), $\mathrm{R}_{\mathrm{f}}$ 0.6 (hexane-EtOAc 1:1). ${ }^{1} \mathrm{H}-\mathrm{NMR}$ : $\delta$ 2.9-3.1 (m, $\mathrm{H}_{\beta, \beta}$ ), 3.71 $\left(\mathrm{s}, \mathrm{CH}_{2} \mathrm{~S}\right) 3.77\left(\mathrm{~s}, \mathrm{OCH}_{3}\right), 4.97\left(\mathrm{~m}, \mathrm{H}_{\alpha}\right), 7.04(\mathrm{~d}, \mathrm{NH}), 7.2-7.3$ $(\mathrm{m}, \mathrm{Ph}), 7.91$ (m, Ar, 2H), 8.25 (m, Ar, 2H); ${ }^{13} \mathrm{C}-\mathrm{NMR}: \delta$ 35.6, 36.9, 52.4, 53.1, 124.0, 127.6, 128.6, 128.9, 129.1, 137.7, 139.3, 150.0, 165.3, 171.2. Anal. Calcd for $\mathrm{C}_{18} \mathrm{H}_{18} \mathrm{~N}_{2} \mathrm{O}_{5} \mathrm{~S}$ : C, 57.74; H, 4.85; N, 7.48. Found: C, 57.71; $\mathrm{H}, 4.83 ; \mathrm{N}, 7.51$.

L-N-(4-Nitrobenzoyl)-S-(trans, trans-farnesyl)cysteine ethyl ester $[(R)$-ethyl 3-((2E,6E)-3,7,11-trimethyldodeca2,6,10-trienylthio)-2-(4-nitrobenzamido)propanoate, L-76]. A solution of $7 \mathrm{~N}$ ammonia in $\mathrm{MeOH}(19 \mathrm{mmol}, 2.7 \mathrm{~mL})$ was added to a solution of L-CysOEt $\mathrm{HCl}(0.56 \mathrm{~g}, 3 \mathrm{mmol})$ in anhydrous $\mathrm{MeOH}(1.5 \mathrm{~mL})$, followed by the addition of farnesyl bromide $(0.94 \mathrm{~g}, 3.3 \mathrm{mmol})$ in one portion at $0^{\circ} \mathrm{C}$. After $30 \mathrm{~min}$ at $0^{\circ} \mathrm{C}$, the mixture was stirred at RT for 30 min, the solvent was removed in vacuum and the residue taken into EtOAc. The precipitated ammonium bromide was centrifuged and the organic phase stirred with $p$-nitrobenzoyl chloride $(0.67 \mathrm{~g}, 3.6 \mathrm{mmol})$ and a solution of $\mathrm{K}_{2} \mathrm{CO}_{3}(0.83 \mathrm{~g}$, $6 \mathrm{mmol})$ in water $(3 \mathrm{~mL})$ with ice cooling for $10 \mathrm{~min}$ and then for $15 \mathrm{~min}$ at RT. The organic phase was separated, dried over $\mathrm{K}_{2} \mathrm{CO}_{3}$ and the solvent evaporated. Column chromatography in DCM afforded $0.51 \mathrm{~g}$ (33\% for 2 steps) of a yellowish solid with m.p. $53-54^{\circ} \mathrm{C},[\alpha]_{\mathrm{D}}{ }^{24}-60.0^{\circ}$ (c 0.06 , acetone), $\mathrm{R}_{\mathrm{f}} 0.75$ (hexane-EtOAc 1:1). ${ }^{1} \mathrm{H}-\mathrm{NMR}: \delta 1.28$ (t, $\left.\mathrm{CH}_{2} \mathrm{CH}_{3}\right), 1.55\left(\mathrm{~s}, 2 \mathrm{CH}_{3}\right), 1.62\left(\mathrm{~s}, 2 \mathrm{CH}_{3}\right), 1.95-2.05$ (m, 4 x CH 2 ), 2.9-3.3 (m, $\left.\mathrm{H}_{\beta, \beta},+\mathrm{CH}_{2} \mathrm{~S}\right), 4.22\left(\mathrm{~s}, \mathrm{OCH}_{2} \mathrm{CH}_{3}\right)$, $4.92\left(\mathrm{~m}, \mathrm{H}_{\alpha}\right), 5.04(\mathrm{~m}, 2 \mathrm{C}=\mathrm{C} \underline{\mathrm{H}}), 5.17(\mathrm{~m}, \mathrm{C}=\mathrm{C} \underline{\mathrm{HCS}}), 7.21$ (d, HN), 7.95 (m, Ar, 2H), 8.22 (m, Ar, 2H). ${ }^{13} \mathrm{C}-\mathrm{NMR}: \delta$ $14.36,16.20,16.35,17.87,25.89,26.61,26.91,30.13,33.42$, $39.82,39.88,52.63,62.31,119.65,123.85,123.99,124.51$, $128.65,131.45,135.59,139.51,140.40,149.95,165.33$, 170.99. Anal. Calcd for $\mathrm{C}_{27} \mathrm{H}_{38} \mathrm{~N}_{2} \mathrm{O}_{5} \mathrm{~S}: \mathrm{C}, 64.51 ; \mathrm{H}, 7.62 ; \mathrm{N}$, 5.57. Found: C, 64.54; H, 7.58; N, 5.64.

L-N-(4-Nitrobenzoyl)-S-(trans, trans-farnesyl)cysteine $i$-propyl ester $[(R)$-isopropyl 3-((2E,6E)-3,7,11-trimethyldodeca-2,6,10-trienylthio)-2-(4-nitrobenzamido)propanoate, L75]. Similar to the synthesis of L-N-(4-nitrobenzoyl)-S(trans,trans-farnesyl)cysteine ethyl ester from CysOPr- $i \mathrm{HCl}$ (0.44 g, $2.2 \mathrm{mmol}), 7 \mathrm{~N}$ ammonia in $\mathrm{MeOH}$ (14 mmol, 2 $\mathrm{mL})$, farnesyl bromide $(0.69 \mathrm{~g}, 2.4 \mathrm{mmol}), p$-nitrobenzoyl chloride $(0.52 \mathrm{~g}, 2.8 \mathrm{mmol})$ and a solution of $\mathrm{K}_{2} \mathrm{CO}_{3}(0.61 \mathrm{~g}$, $4.4 \mathrm{mmol})$ in water $(3 \mathrm{~mL})$. A yield of $0.33 \mathrm{~g}(29 \%$ for 2 steps) of a yellowish solid with m.p. $60-61^{\circ} \mathrm{C},[\alpha]_{\mathrm{D}}{ }^{24}-71.7^{\circ}$ (c 0.05 , acetone), $\mathrm{R}_{\mathrm{f}} 0.8$ (hexane-EtOAc 1:1). ${ }^{1} \mathrm{H}$ NMR: 1.26 [dd, 1.2, $\left.6.3 \mathrm{~Hz}, \mathrm{OCH}\left(\mathrm{CH}_{3}\right)_{2}\right], 1.54\left(\mathrm{~s}, 2 \mathrm{CH}_{3}\right), 1.62(\mathrm{~s}, 2$ $\mathrm{CH}_{3}$ ), 1.9-2.1 (m, 4 x CH $\mathrm{CH}_{2}$ ), 2.93 (dd, 6.0, $13.8 \mathrm{~Hz}, \mathrm{H}_{\beta}$ ), 3.05$3.30\left(\mathrm{~m}, \mathrm{H}_{\beta},+\mathrm{CH}_{2} \mathrm{~S}\right), 4.89\left(\mathrm{~m}, \mathrm{H}_{\alpha}\right), 5.0-5.2[\mathrm{~m}, 2 \mathrm{C}=\mathrm{CH}+$ $\left.\mathrm{C}=\mathrm{C} \underline{\mathrm{HCS}}+\mathrm{OC} \underline{\mathrm{H}}\left(\mathrm{CH}_{3}\right)_{2}\right], 7.18(\mathrm{~d}, \mathrm{HN}), 7.95(\mathrm{~m}, \mathrm{Ar}, \overline{2 \mathrm{H}})$, 8.22 (m, Ar, 2H). ${ }^{13} \mathrm{C}$ NMR: $\delta 16.20,16.36,21.92,21.95$, 25.88, 26.63, 26.90, 30.18, 33.46, 39.82, 39.87, 52.75, 70.28, 
$119.68,123.84,123.98,124.51,128.61,131.44,135.58$, $139.57,140.31,149.95,165.29,170.49$. Anal. Calcd for $\mathrm{C}_{28} \mathrm{H}_{40} \mathrm{~N}_{2} \mathrm{O}_{5} \mathrm{~S}: \mathrm{C}, 65.09 ; \mathrm{H}, 7.80 ; \mathrm{N}, 5.42$. Found: $\mathrm{C}, 65.31$; $\mathrm{H}, 7.77$; N, 5.51.

$\mathrm{N}$-(4-Nitrobenzoylglycyl)-S-(trans, trans-farnesyl)cysteine methyl ester [RD-18]. 1N solution of N,N'-dicyclohexylcarbodiimide in DCM (1.5 mL, $1.5 \mathrm{mmol})$ was added to a stirred suspension of 4-nitrohippuric acid (336 mg, 1.5 $\mathrm{mmol}$ ) and FarCysOMe (488 mg, $1.44 \mathrm{mmol}$ ) in anhydrous DCM $(10 \mathrm{~mL})$ and the mixture was kept at RT for one day. The solvent was evaporated in vacuum and the solid residue crystallized from hot methanol (18-1, $418 \mathrm{mg}, 53 \%)$; mp $119-120^{\circ} \mathrm{C} ; \mathrm{R}_{\mathrm{f}} 0.4$ (hexane-EtOAc). The mother liquor was evaporated in vacuum, the residue dissolved in DCM and separated on a column using a gradient DCM-EtOAc 15:1 to $7: 1$. Crystallization from methanol with activated carbon gave $(18-2,210 \mathrm{~g}, 27 \%) ; \mathrm{mp} 118-120^{\circ} \mathrm{C} ;[\alpha]_{\mathrm{D}}{ }^{20}=-20.9^{\circ}$ (c=0.03, acetone); IR (KBr) NH (3300), $\mathrm{C}=\mathrm{O}$ (1750), $\mathrm{O}=\mathrm{CN}$ (1650), $\mathrm{NO}_{2}(1340) \mathrm{cm}^{-1} .{ }^{1} \mathrm{H}-\mathrm{NMR}: \delta 1.55-1.60\left(\mathrm{~s}, \mathrm{CH}_{3}\right)$, 1.61-1.66 (s, $\left.\mathrm{CH}_{3}\right), 1.59\left(\mathrm{~s}, \mathrm{CH}_{3}\right), 1.66\left(\mathrm{~s}, \mathrm{CH}_{3}\right), 1.93-2.10$ $\left(\mathrm{m}, 4 \times \mathrm{CH}_{2}\right), 2.82-2.90\left(\mathrm{H}_{\beta}\right)$ and $2.95-3.01\left(\mathrm{H}_{\beta}\right)(\mathrm{dd}, \mathrm{S}-\mathrm{C}-\mathrm{H}$ $J=7.5 \mathrm{~Hz}), 3.01-3.24\left(\mathrm{t}, \mathrm{CH}_{2} \mathrm{~S}, J=7.2 \mathrm{~Hz}\right), 3.77\left(\mathrm{~s}, \mathrm{OCH}_{3}\right)$, 4.276 (d, 2 x), 4.78-4.84 (t, $\mathrm{HC}^{-N_{2}}$ ), 5.05-5.09 (m, 2 x $\mathrm{C}=\mathrm{CH}), 5.15-5.20(\mathrm{~m}, \mathrm{C}=\mathrm{C} \underline{\mathrm{HCS}}, J=1.2 \mathrm{H}), 7.21-7.27(\mathrm{~d}, \mathrm{HN}$, $J=10.2 \mathrm{~Hz}), 7.58(\mathrm{~s}, \mathrm{NH}), 7.98(\mathrm{~m}, \mathrm{Ar}, 2 \mathrm{H}, 2 \mathrm{H}), 8.27$ (m, Ar, $2 \mathrm{H}, 2 \mathrm{H}) .{ }^{13} \mathrm{C}-\mathrm{NMR}: \delta 16.27,16.39,17.95,25.97,26.64$, $26.93,30.07,33.28,39.87,39.27,43.71,52.25,53.08,119.6$, 123.8, 124.0, 124.5, 128.7, 131.6, 135.7, 139.2, 140.5, 149.9, 165.8, 168.9, 171.3. Anal. Calcd for $\mathrm{C}_{28} \mathrm{H}_{39} \mathrm{~N}_{3} \mathrm{O}_{6} \mathrm{~S}: \mathrm{C}, 61.63$; H, 7.20; N, 7.70. Found: C, 61.48; H, 7.02; N, 7.59.

L-N-(4-Nitrobenzoyl)-alanine methyl ester [(S)-methyl 2(4-nitrobenzamido)propanoate, L-111]. TMS-Cl (3 mL, 23.5 mmol) was added to a suspension of L-Ala $(891 \mathrm{mg}, 10$ $\mathrm{mmol})$ in $\mathrm{MeOH}(17 \mathrm{~mL})$ at RT and left overnight. After removal of the solvent, solid L-Ala methyl ester hydrochloride was stirred with $p$-nitrobenzoyl chloride $(2.04 \mathrm{~g}, 11$ $\mathrm{mmol}), \mathrm{K}_{2} \mathrm{CO}_{3}(4.15 \mathrm{~g}, 30 \mathrm{mmol})$, water $(15 \mathrm{~mL})$ and EtOAc $(35 \mathrm{~mL})$ for $1 \mathrm{~h}$. The organic layer was dried over $\mathrm{Na}_{2} \mathrm{SO}_{4}$, evaporated and crystallized from hot $\mathrm{MeOH}(10 \mathrm{~mL})$. A pale yellow solid (1.67 $\mathrm{g}, 66 \%$ ) with $\mathrm{R}_{\mathrm{f}} 0.8$ (hexane-EtOAc 1:2) and m.p. $134-135^{\circ} \mathrm{C},[\alpha]_{\mathrm{D}}{ }^{25}+42.1$ (c $0.04, \mathrm{CHCl}_{3}$ ) was obtained. Anal. Calcd for $\mathrm{C}_{11} \mathrm{H}_{12} \mathrm{~N}_{2} \mathrm{O}_{5}$ : C, 52.28; H, 4.80; N, 11.11. Found: C, 52.65; H, 4.76; N, 11.02 .

\section{Sequencing and Identification of Esterase from Porcine Liver}

We previously reported the purification of a single esterase of about $57 \mathrm{kDa}$ (SDS-PAGE) to $62 \mathrm{kDa}$ (calculated) from porcine liver that hydrolyzes trans, transfarnesylated cysteine methyl ester substrates [14]. EPL obtained from Sigma-Aldrich (St. Louis, MO) was found to hydrolyze polyisoprenylated substrates while AChE and $\mathrm{BChE}$ did not. With limited information regarding the purity, molecular weight and amino acid sequence identity of the Sigma-Aldrich EPL, we sought to analyze it for three reasons; (1) assess its purity, (2) compare its molecular weight with the PMPMEase we previously purified from porcine liver and (3) if identical, switch to the more cost-effective commercially available enzyme. To achieve this, an aliquot of the enzyme ( $1 \mu \mathrm{g})$ was analyzed by SDSPAGE on an $8 \%$ gel followed by EZ-Blue colloidal coomas- sie blue staining. The protein band was excised and submitted to ProtTech (Eagleville, PA) for trypsin digestion and LC-MS/MS sequencing and identification. An aliquot of EPL was washed on C-4 Q-tips and mixed with sinapinic acid matrix followed by analyses on the Applied Biosystems 4800 Mass Spectrometer in linear mode (Applied Biosystems, Framingham, MA).

\section{Enzyme Assays}

Each substrate (1 mM final concentration) was incubated with either PMPMEase (1-10 $\mu \mathrm{g}$ depending on substrate), acetylcholinesterase $(10 \mu \mathrm{g})$ or butyrylcholinesterase $(10 \mu \mathrm{g})$ in $100 \mathrm{mM}$ Tris- $\mathrm{HCl}, \mathrm{pH} 7.4$ containing $5 \%$ DMSO at $37^{\circ} \mathrm{C}$ (100 $\mu \mathrm{l}$ total volume). Michaelis-Menten kinetics analysis was conducted under identical conditions except with varying concentrations of the different substrates and EPL ( 0.1 to $5 \mu \mathrm{g} / \mathrm{per}$ assay depending on substrate). The reactions were stopped and analyzed as previously described $[14,24]$ with UV-detection at $260 \mathrm{~nm}$ (for the para-nitrobenzoyl susbtrates) or $214 \mathrm{~nm}$ for BzGFCM, HCFCM and L-69 (D-AFCM). Aliquots of the substrates were dissolved in a $50 \%(\mathrm{v} / \mathrm{v})$ mixture of methanol in water containing $0.05 \mathrm{M}$ $\mathrm{NaOH}$ and incubated at $37^{\circ} \mathrm{C}$ for $1 \mathrm{~h}$ to hydrolyze the esters to the corresponding acid. These were then used as HPLC retention time markers as well as to generate calibration plots for data analysis. The $K_{\mathrm{M}}$ and $\mathrm{Vmax}$ values were obtained by applying Michaelis-Menten kinetics analysis on the data using the GraphPad Prism software.

\section{Modeling of S-Alkylated Cysteinyl-Ester Substrates}

The $\mathrm{p} K_{\mathrm{M}}$ for twelve of the substrates (Table 1) were used in Comparative Molecular Field Analysis (CoMFA). The initial geometries were generated using SKETCH/Sybyl 8.0 (Tripos Associates Inc., St. Louis, MO) and the side-chains were set to the extended conformations. The Sybyl software was run under the LINUX operating system (Fedora 8.0) and the lowest energy (minimized) conformations were obtained under vacuum using the conjugate gradient method with $0.001 \mathrm{kcal} /(\mathrm{mol} \AA)$ ) gradient termination option and 5000 maximum iterations, using MMFF94s force field and MMFF94 charges.

Several alignment hypotheses were investigated in the absence of a known pharmacophore for this group of compounds. The alignment that led to the highest cross-validated $\left(\mathrm{q}^{2}\right)$ value was selected for the development of the final model. The minimized structure of one of the most active compounds (L-75) was used as the template. The rest of the compounds were aligned using the SYBYL "database alignment" function and a common set of 6 atoms from the ester carbonyl carbon to the first double-bond carbon of the side chain (C11-C10-C15-S16-C17-C18 shown in L-75 in Table 1).

\section{RESULTS}

\section{Synthesis}

The general procedures for the synthesis of the substrates are shown in Scheme 1. Proton and ${ }^{13} \mathrm{C}$ NMR, elemental analysis and reversed-phase high performance liquid chromatography confirmed the identities of the synthesized compounds and the over $95 \%$ purity of the compounds. 
Table 1. Michaelis-Menten Kinetic Data for the Hydrolysis of Carboxyl Esters by PMPMEase. The pK $K_{M}$ Values for the Training Dataset used in the Molecular Modeling are Shown in Square Brackets

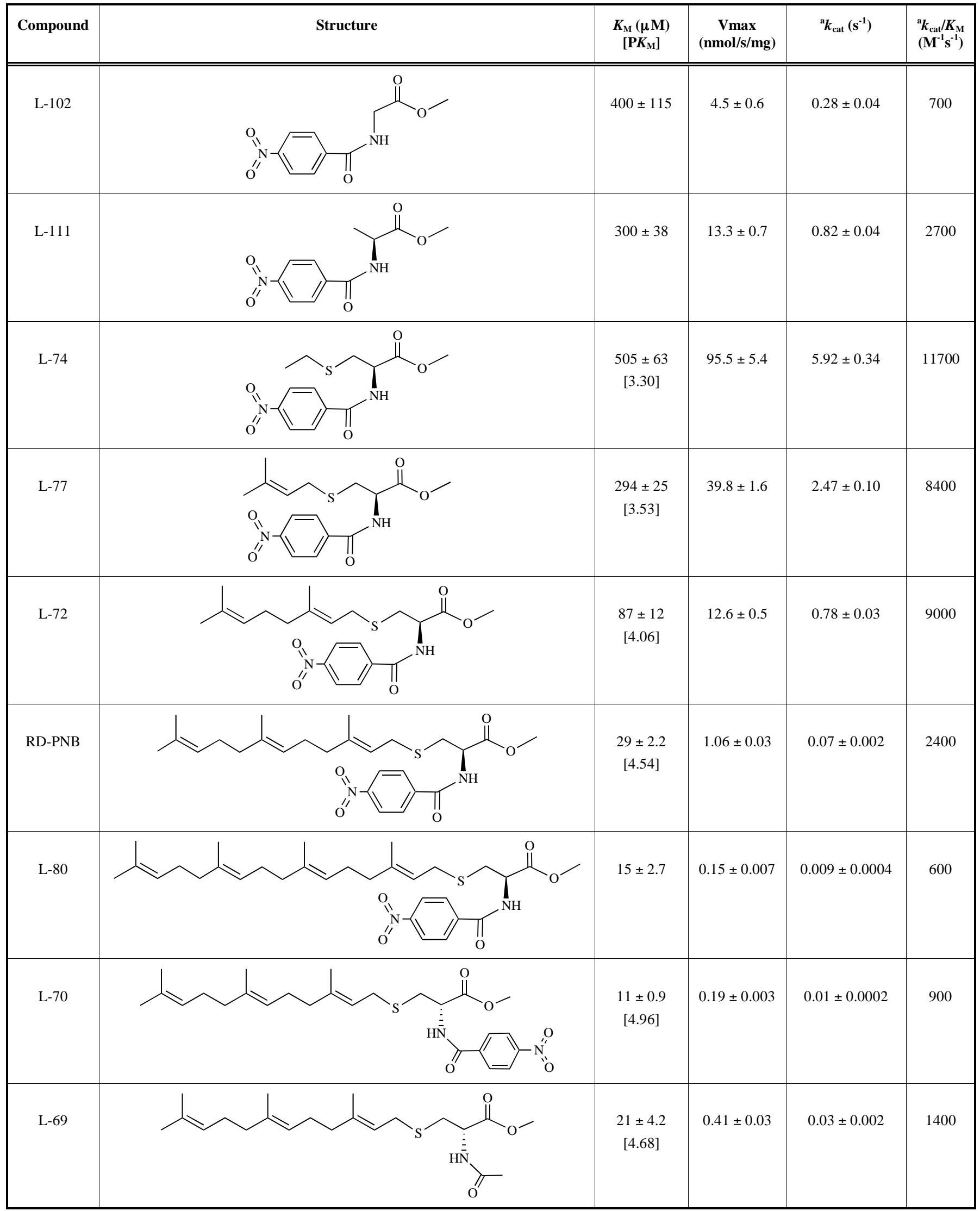


Table 1. contd....

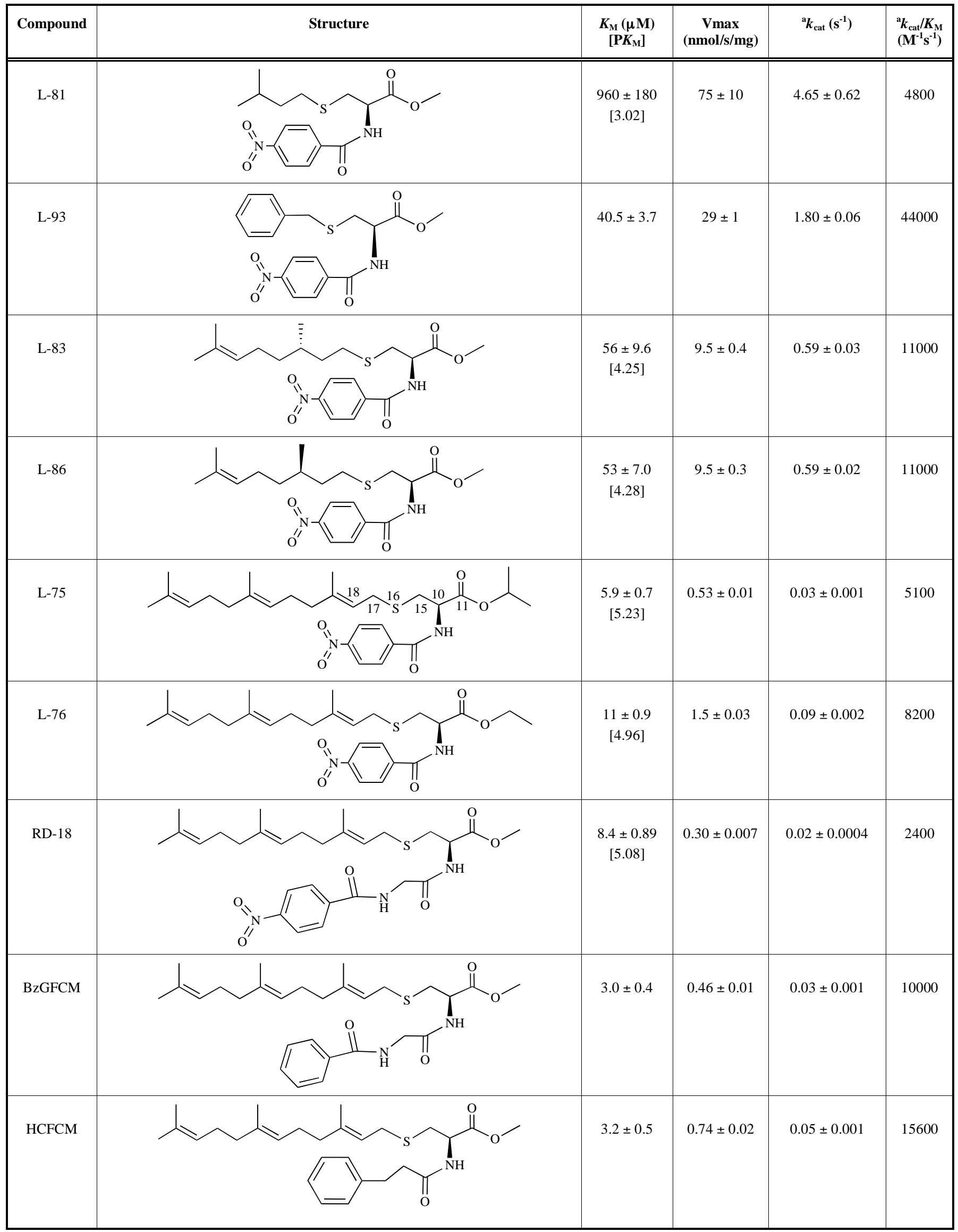




\section{EPL is the Same Protein as PMPMEase}

SDS-PAGE analysis of EPL revealed a single major protein band of about $60.5 \mathrm{kDa}$ (Fig. 1A). Linear mode MS analysis showed two peaks corresponding to the $[\mathrm{M}+\mathrm{H}]^{+}$and $[\mathrm{M}+2 \mathrm{H}]^{2+}$ molecular ions with $\mathrm{M} / \mathrm{Z}$ values of 61,200 and 30,600, respectively (Fig. 1B). LC-MS/MS analysis of the coomassie band yielded the sequences of over twenty-five tryptic peptides which were used to identify a single protein with greater than $98 \%$ certainty. EPL was identified as EST1_PIG Liver carboxylesterase precursor, Proline-betanaphthylamidase, Retinyl ester hydrolase or carboxylesterase precursor (Sus scrofa) with a calculated molecular weight of 62621.95. EPL is thus the same protein as PMPMEase that was previously purified and identified as carboxylesterase precursor (Sus scrofa) [14].

\section{S-Alkylated Substrates are Hydrolyzed by PMPMEase but not $\mathrm{AChE}$ and $\mathrm{BChE}$}

When the substrates were analyzed for hydrolysis by PMPMEase, AChE and BChE, all of the substrates were significantly hydrolyzed by PMPMEase. Little or no hydrolysis was observed with AChE and BChE (Fig. 2). The S-substitutions had a strong effect on the degree of hydrolysis by PMPMEase and the cholinesterase enzymes. The all trans-geranylgeranylated and the trans,trans-farnesylated substrates (L-69, L-70, L-75, L-76, L-80 and RD-PNB) were all very resistant to cholinesterase hydrolysis (Fig. 2). Substrates with significantly reduced side-chains (L-74, L-102 and L-111) were more susceptible to hydrolysis by acetyl- and butyrylcholinesterases (Fig. 2). A time-dependent study further revealed more rapid hydrolysis of the S-prenylated (L-77) and S-geranylated (L-72) substrates over the trans,trans-farnesylated and S-all trans-geranylgeranylated substrates by PMPMEase (Fig. 3).

\section{Michaelis-Menten Kinetics Analysis}

We hypothesized that if polyisoprenylated proteins are the substrates for EPL/PMPMEase, then substrates with trans, trans-farnesyl or all trans-geranylgeranyl moieties will display the lowest $K_{\mathrm{M}}$ values. The analysis revealed the typi- cal concentration-dependent effects (Fig. 4). As expected, the substrates with small S-substituents (e.g. ethyl and prenyl) displayed the least affinity while the trans,transfarnesyl and all trans-geranylgeranyl substrates had the highest affinities (Fig. 4). The $K_{\mathrm{M}}$ values ranged from $960 \pm$ $180 \mu \mathrm{M}$ (L-81, L-alanine) to $3.0 \pm 0.4 \mu \mathrm{M}$ (BzGFCM), a 320 -fold increase in affinity (Table $\mathbf{1}$ ).

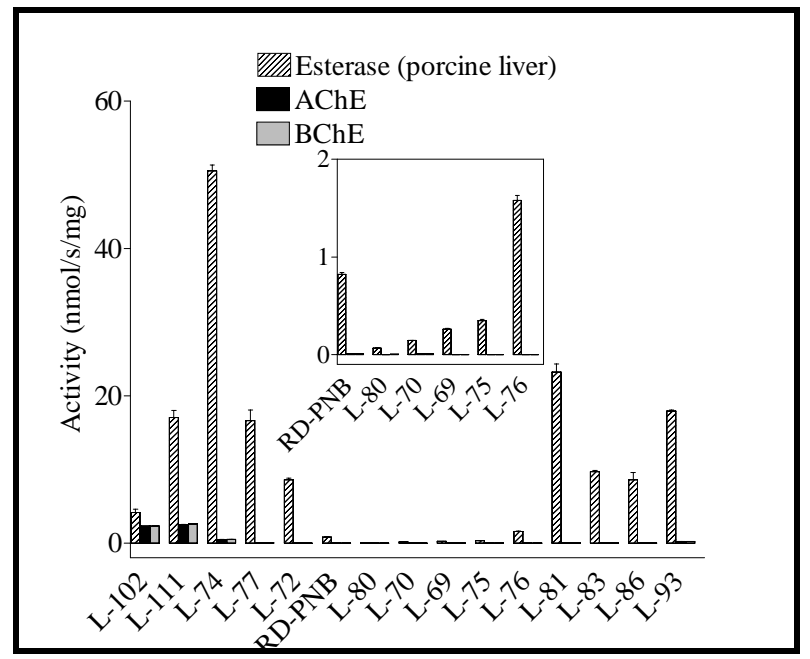

Fig. (2). Relative substrate hydrolysis by PMPMEase, acetyl- and butyrylcholinesterases. Each substrate was incubated with the different enzymes as described in the procedures section. These were then analyzed for the amounts of products formed. The specific activities were then computed based on the amounts of enzyme used and the duration of the incubation to determine the specific activity. The results are the means $\pm \operatorname{SEM}(n=3)$.

Use of the D- in place of L-cysteine did not prevent hydrolysis by PMPMEase. Instead, the affinity was increased by almost 3-fold as shown by the $K_{\mathrm{M}}$ values for the Lcysteine analog, RD-PNB $(29 \pm 2.2 \mu \mathrm{M})$ compared to the Dcysteine analog, L-70 $(11 \pm 0.9 \mu \mathrm{M})$. Larger O-alkyl
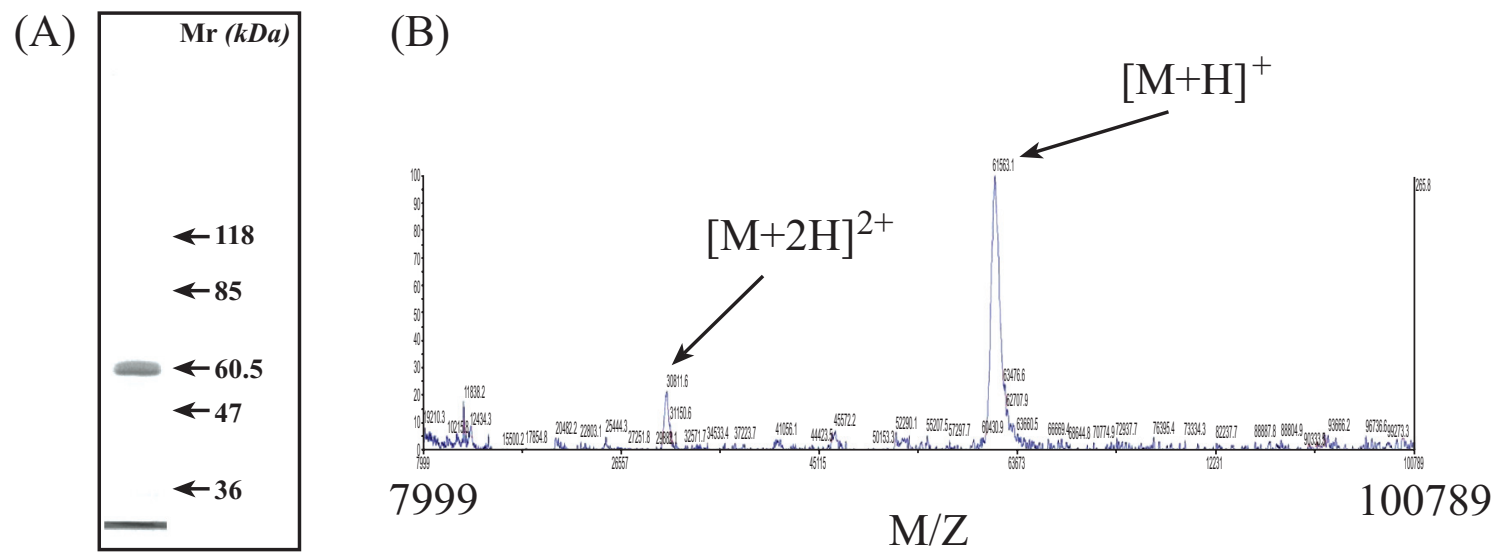

Fig. (1). SDS-PAGE and mass spectrometry analyses reveal EPL is the same protein as PMPMEase. (A) A $1 \mu \mathrm{g}$ aliquot of EPL was boiled in SDS-PAGE sample buffer and subjected to electrophoresis on an $8 \%$ polyacrylamide gel followed by EZ-Blue colloidal coomassie blue staining. The molecular weight of the protein band was determined based on its migration relative to the Fisher's EZ-Run pre-stained molecular weight protein markers shown in kilodaltons ( $\beta$-galactosidase, 118.0; bovine serum albumin 85.0; ovalbumin, 47.0 and lactate dehydrogenase, 36.0). (B) MALDI-TOF analysis of an aliquot of EPL using the Applied Biosystems 4800 mass spectrometer. The protein peaks represent the $[\mathrm{M}+\mathrm{H}]^{+}(\mathrm{M} / \mathrm{Z}=61,200)$ and $[\mathrm{M}+2 \mathrm{H}]^{2+}(\mathrm{M} / \mathrm{Z}=30,600)$ molecular ions of EPL. 
substituents also increased the affinity towards the esterase as O-ethyl (L-76) and O-isopropyl (L-75) had lower $K_{\mathrm{M}}$ values $(11 \pm 0.9$ and $5.9 \pm 0.66 \mu \mathrm{M}$, respectively) when compared to RD-PNB $(29 \pm 2.2 \mu \mathrm{M})$.
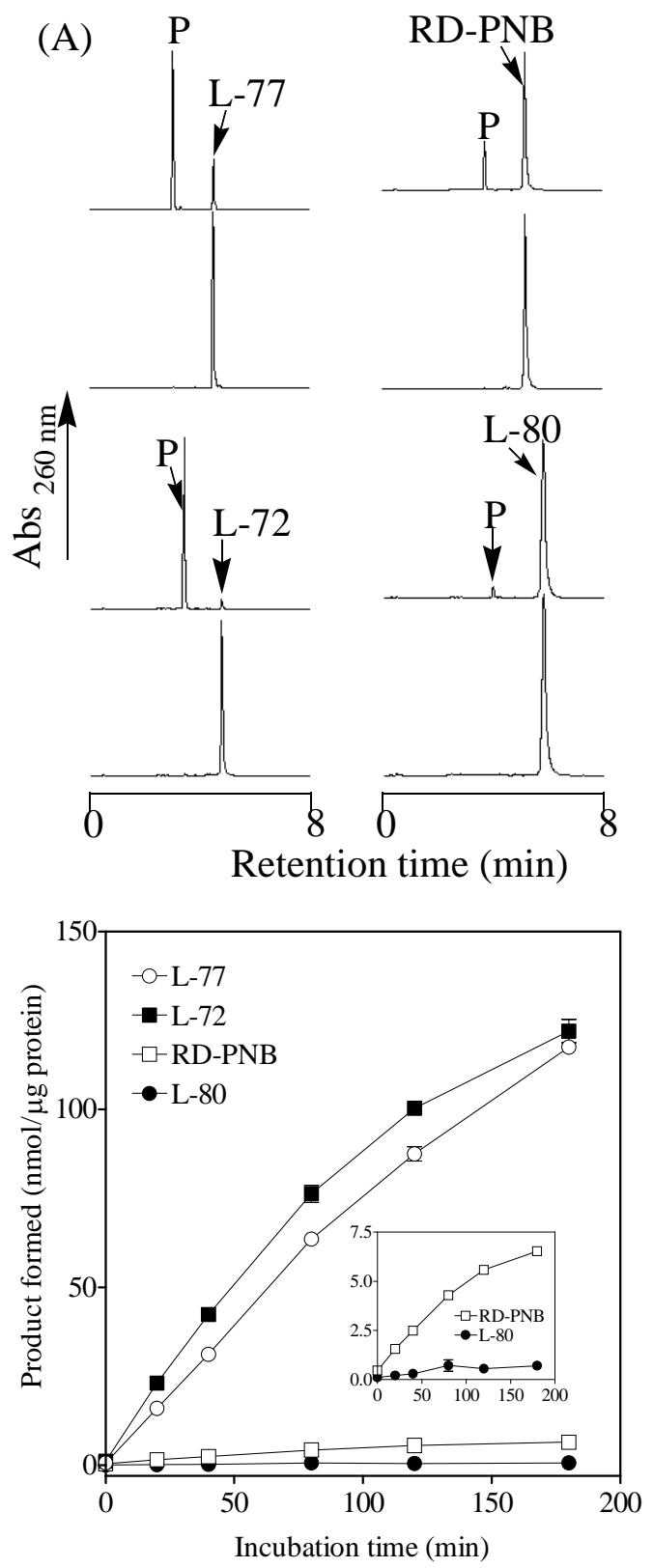

Fig. (3). Time-dependent hydrolysis of selected S-alkylated substrates. An S-prenylated (L-77), S-trans geranylated (L-72), $\mathrm{S}$-trans, trans-farnesylated (RD-PNB) and S-all trans-geranylgeranylated (L-80) substrates at $1 \mathrm{mM}$ concentrations were incubated with either 0.25 or $1 \mu \mathrm{g}$ of EPL. At the various time intervals, the reactions were stopped and analyzed as described in the procedures section. The amounts of enzyme used and the amounts of products formed were used to compute the relative amounts of products formed. Panel A: Reversed-phase HPLC chromatograms showing the substrate hydrolysis to products $(\mathrm{P})$ by $5 \mu \mathrm{g}$ PMPMEase following $160 \mathrm{~min}$ incubation (upper chromatograms) compared to the respective substrates before incubation (lower chromatograms). Panel B: Time-dependent hydrolysis of the selected substrates. The results are the means \pm SEM $(n=3)$.
Bond saturation on the S-substituents yielded ambiguous results as shown by the $K_{\mathrm{M}}$ values for L-77 $(290 \pm 25 \mu \mathrm{M})$ versus L-81 $(960 \pm 180 \mu \mathrm{M})$ as well as L-72 $(87 \pm 12 \mu \mathrm{M})$ compared to L-83 $(56 \pm 9.6 \mu \mathrm{M})$ and $\mathrm{L}-86(53 \pm 7.0 \mu \mathrm{M})$. The S-benzyl analog (L-93) displayed a biphasic character in which the typical Michaelis-Menten type saturation kinetics was observed at concentrations lower than $125 \mu \mathrm{M}$ but an inhibitory effect was observed at higher concentrations. A limited concentration range was then used to obtain the $K_{\mathrm{M}}$ of $40.5 \pm 3.7$. Because it is difficult to determine the substrate concentration at which the inhibitory effects begin, the true $K_{\mathrm{M}}$ value for L-93 may be significantly higher than the obtained value. This may have resulted in the much larger $k_{\text {cat }} / K_{\mathrm{M}}$ value for L-93 of 44,000 .

For the S-alkylated $p$-nitrobenzoyl-L-cysteine methylester substrates, the Vmax values increased inversely with the $K_{\mathrm{M}}$ values and decreased S-alkyl substituent sizes. The Vmax values for the substrates lacking the sulfur atom (L102 and L-111) did not fit this general pattern as might have been predicted from the large $K_{\mathrm{M}}$ values. Decreasing the alkyl chain from the 20-carbon all trans-geranylgeranyl through trans,trans-farnesyl, trans-geranyl, prenyl to ethyl resulted in at least 7-, 80-, 260- and 600-fold increases in the relative hydrolysis rates (Table $\mathbf{1}$ ).

\section{Modeling of S-alkyl,N-acyl Cysteinyl-Ester Substrates}

CoMFA is widely used to study the correlation of biological activities with the structural properties of ligands. It samples the steric and electrostatic fields around a set of ligands and correlates them to the experimental binding affinities. Evaluation of the alignment maps (Fig. (5), top panels) shows that the spatial location of the $p$-nitrobenzoyl moiety in the S-isomers compared to the R-isomers (Fig. (5) bottom left panel) is in the opposite direction. Also, in the extended side chains of compounds L-83 and L-86, the C-18 saturated analogs, were placed away from those of the template compound as depicted with L-86 and L-75 (Fig. (5) bottom right panel). The initial Leave-One-Out crossvalidated partial least squares (PLS) analysis of the aligned dataset yielded a cross-validated $\mathrm{q}^{2}$ of 0.863 with a standard error of 0.365 at component 5 . The final quantitative structure-activity relationship (QSAR) model yielded an $\mathrm{R}^{2}$ of 0.995 with a standard error of 0.071 . The statistical data of the PLS analysis are summarized in Table $\mathbf{2}$ and a plot of the predicted against experimental binding affinities $\left(\mathrm{p} K_{\mathrm{M}}\right)$ is shown in Fig. (6). A predicted $\mathrm{p} K_{\mathrm{M}}$ of 4.72 was obtained for L-80 that was not included in the training set, compared to its experimental value of 4.82 .

CoMFA contour maps are useful in providing visual displays of areas where the steric and electrostatic fields significantly affect binding affinities. Fig. (7) (top left panel) shows the contour map of the model with the polyisoprenyl group of the template compound, L-75, interacting with the green region of the final CoMFA fields. On the other hand, L-81, (Fig. (7) top right panel), does not extend to interact with this region. The insufficient interactions (Fig. (7) bottom left panel) are observed with other substrates with smaller Ssubstituents than L-75. The S-alkyl side chains of L-83 and L-86 have C-18-saturated carbons which result in them being placed away from the equivalent side chain of the template molecule (Fig. (7) bottom right panel), preventing them from 

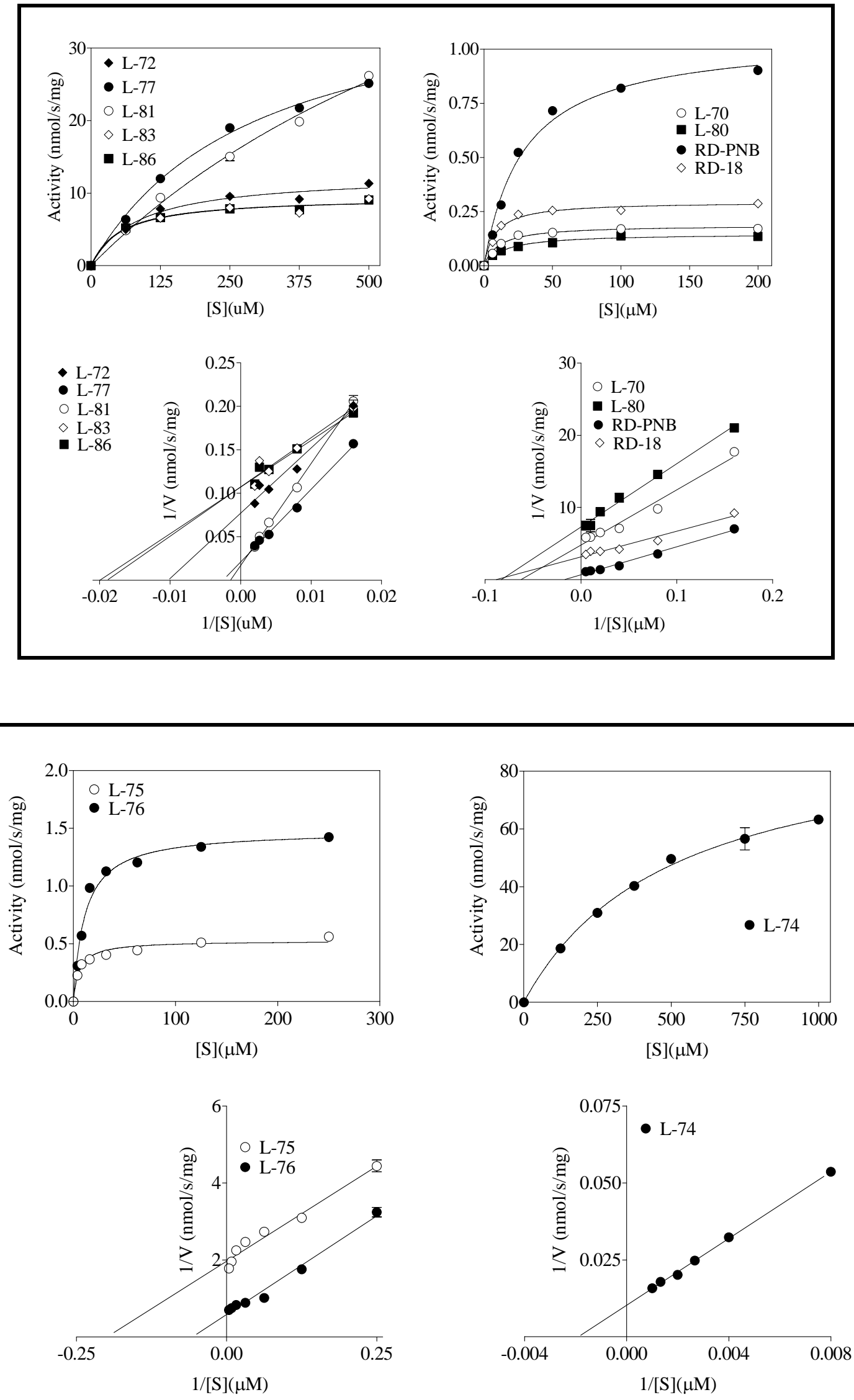

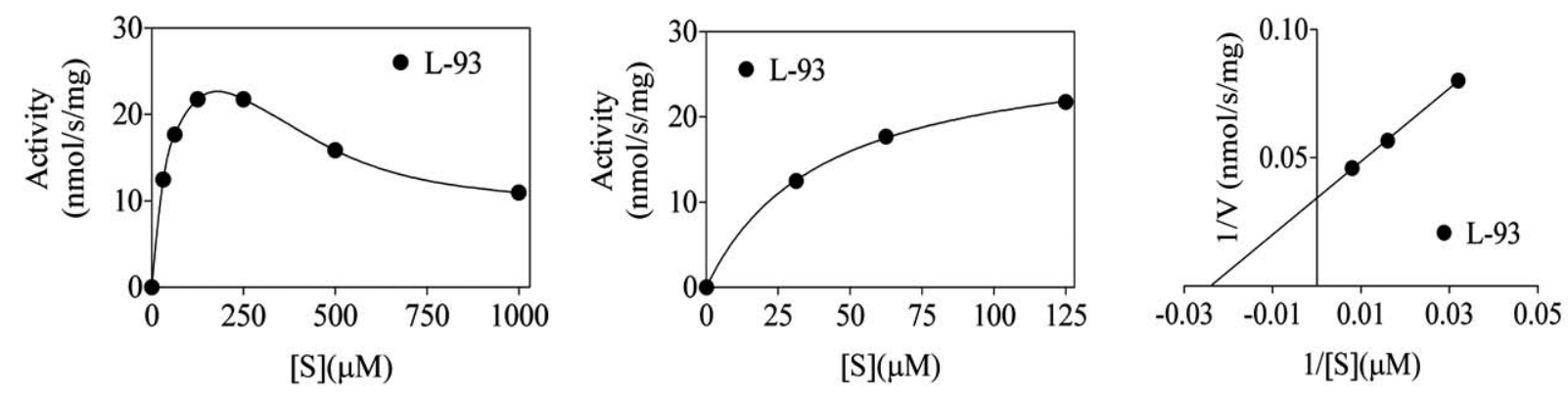

Fig. (4). Michaelis-Menten and Double-reciprocal analysis of ester substrates hydrolysis by PMPMEase. PMPMEase (0.1 to $5 \mu \mathrm{g}$ depending substrate) was incubated with varying concentrations of each substrate. The reactions were stopped and analyzed by reversed phase-HPLC as described in the procedures section. The specific activities were calculated and used to determine the $K_{\mathrm{M}}$ and Vmax values using the GraphPad Prism software. The results are the means \pm SEM $(n=3)$.
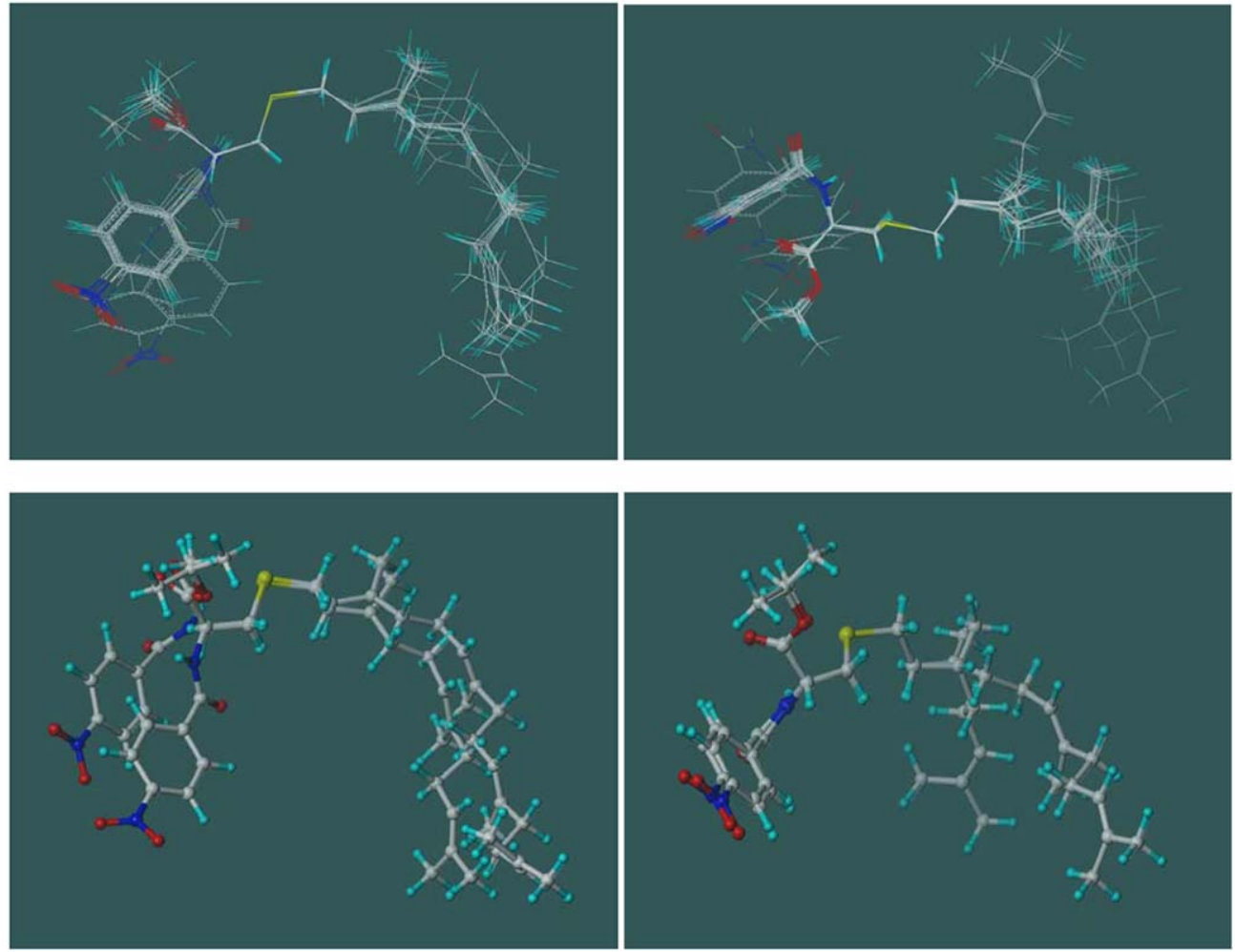

Fig. (5). Two views of the alignment of all molecules in the training set (top panels). The alignment of L-70 and L-75 (bottom left panel) and L-86 and L-75 (bottom right panel).

interacting with the green contour region. The steric contour map also shows a smaller green region adjacent to the ester carbonyl carbon $(\mathrm{C} 11)$. The contribution of the electrostatic field is low (28\%) and does not provide an interpretive result.

\section{DISCUSSION}

Although a single polyisoprenylated substratemetabolizing esterase was previously purified, sequenced and identified from porcine liver [14], its selectivity towards small molecule trans,trans-farnesylated and S-all trans- 
geranylgeranylated cysteine methyl esters that would be indicative of its status as the polyisoprenylation-dependent esterase has not been demonstrated. Such an esterase would have higher affinities (lower $K_{\mathrm{M}}$ values) for S-trans,transfarnesylated and S-all trans-geranylgeranylated cysteine methyl esters than for other S-alkyl substituents. The significantly higher affinity of the enzyme towards the Strans, trans-farnesylated and S-all trans-geranylgeranylated cysteine methyl esters indicates that polyisoprenylated proteins are most likely the endogenous substrates of the enzyme. Since the substrates with progressively shorter Salkyl groups displayed increasingly lower affinity towards the enzyme, it can be inferred, as shown in the modeling contour maps, that the polyisoprenyl moiety is pivotal in the active site binding interactions. Studies with synthetic mimics of the carboxyl terminals of polyisoprenylated proteins revealed similar affinities towards PPMTase [2528]. The strong correlation between the experimental and predicted binding affinities affirms the principal finding that S-trans, trans-farnesylation and S-all trans-geranylgerannylation constitute high affinity elements of a substrate towards the enzyme.

Table 2. Summary of the CoMFA Results

\begin{tabular}{|c|c|}
\hline PLS Statistics & \\
\hline \hline Cross-validated $\mathrm{q}^{2}$ & 0.863 \\
\hline Standard error of prediction (Cross-validated) & 0.365 (at comp 5) \\
\hline$\%$ Variance & 0.994 (at comp 5) \\
\hline $\mathrm{R}^{2}$ & 0.995 \\
\hline Standard error of estimate (Non-cross validated) & 0.071 \\
\hline F values (n1=5, n2=6) & 227 \\
\hline PLS components & 5 \\
\hline Field contributions & 0.722 \\
\hline Steric & 0.278 \\
\hline Electrostatic & \\
\hline
\end{tabular}

The low turnover rates for the S-trans,trans-farnesylated and S-all trans-geranylgeranylated cysteine methyl ester substrates compared to the short S-alkyl substrates is not unexpected. As the modeling contour maps reveal, the strong binding site is beyond the reach of short S-alkyl chains compared to the S-tran,trans-farnesyl and S-all transgeranylgeranyl moieties. The products from substrates bearing the latter two therefore interact more strongly with the enzyme resulting in slower dissociations than the short chain S-alkyls, thereby prolonging enzyme recovery times resulting in lower turnover rates. Bond saturation around C-18 as in compounds L-83 and L-86 compared to L-72 and L-81 compared to L-77 had opposing effects on the affinity towards the enzyme. This suggests that a trigonal planar structure may be preferred at this position. The tetrahedral structure arising from the saturation, though negatively impacting the affinity (L-81), resulted in a different spatial orientation of the additional isoprene in L-83 and L-86 as the modeling depicts. This might have resulted in affinity contacts for L83 and L-86 with the enzyme molecule that more than com- pensates for the diminished affinity displayed in L-81. This is corroborated by the aligned structures which reveal the profiles of the S-alkyl portions of L-83 and L-86 slightly turning at a different trajectory at the saturation point from those of L-72 and the other molecules. The alignment from the susceptible carbonyl carbon through to the first two carbon atoms of the S-alkyl substituents explains why the D-cysteine analogs were surprisingly hydrolyzed by the enzyme. It appears from these that the spatial orientation of the $\mathrm{N}$-substituents is the most altered while the S-alkyl groups that are important for affinity and the ester group that is the target for hydrolysis are not affected by the switch from L- to D-cysteine. The much higher $k_{\text {cat }} / K_{\mathrm{M}}$ value for the S-benzyl substrate (L-93) is most likely a result of the inhibitory effect (on the $K_{\mathrm{M}}$ ) that is more obvious at high concentrations. Although the lower concentrations were used in the Michaelis-Menten kinetics computations, the inhibitory effects may have been large enough to significantly suppress the $K_{\mathrm{M}}$. The $K_{\mathrm{M}}$ as shown is at least 2 to 24 -fold smaller than the $K_{\mathrm{M}}$ values for the comparably sized L-72, L-81 and L-77. A lesser pronounced inhibitory effect at concentrations over $200 \mu \mathrm{M}$ was also observed for HCFCM and BzGFCM. Unlike the other substrates, the latter two compounds and L93 are characterized by a planar unsubstituted benzene ring in the molecule which may fit into a fissure-like allosteric inhibitory site on the enzyme molecule.

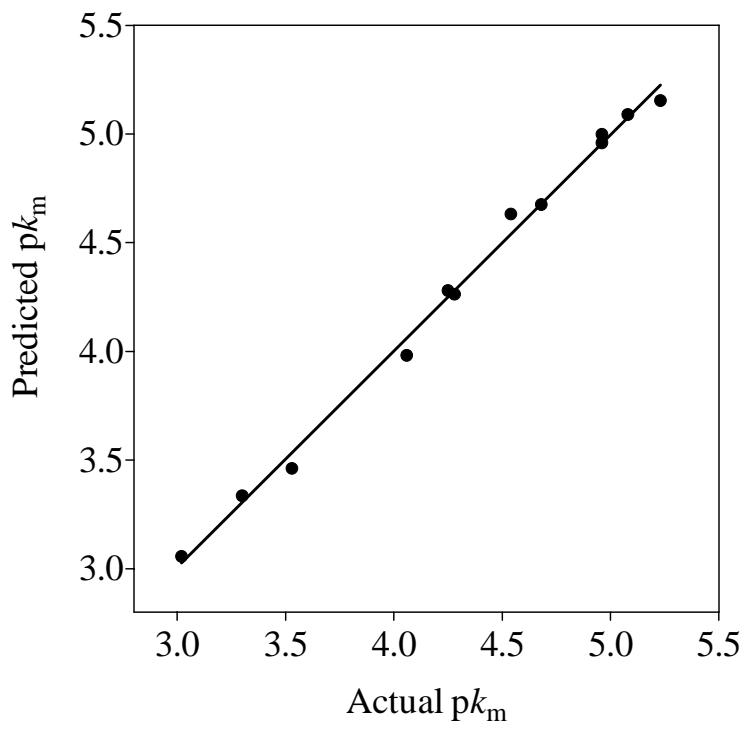

Fig. (6). Predicted (y-axis) versus experimental logs of the binding affinities $\left(\mathrm{p} K_{\mathrm{M}}, \mathrm{X}\right.$-axis). The experimental $\mathrm{p} K_{\mathrm{M}}$ for one compound was removed from the model and the model was then used to obtain the predicted $\mathrm{p} K_{\mathrm{M}}$ for that substrate. This process was repeated for the other substrates to obtain the respective $\mathrm{p} K_{\mathrm{M}}$ values. The results of the predicted $\mathrm{p} K_{\mathrm{M}}$ values were then plotted against the experimentally-derived binding affinities.

The wide distribution of PMPMEase in various organs [24] is in line with the highly ubiquitous nature of their putative polyisoprenylated proteins. Although these enzymes are yet to be characterized, various esterases with sequence identities to PMPMEase in excess of $70 \%$ have been reported from the intestines [29], human brain [30], human alveolar macrophages [31], human monocytes and macrophages [31, 32]. 

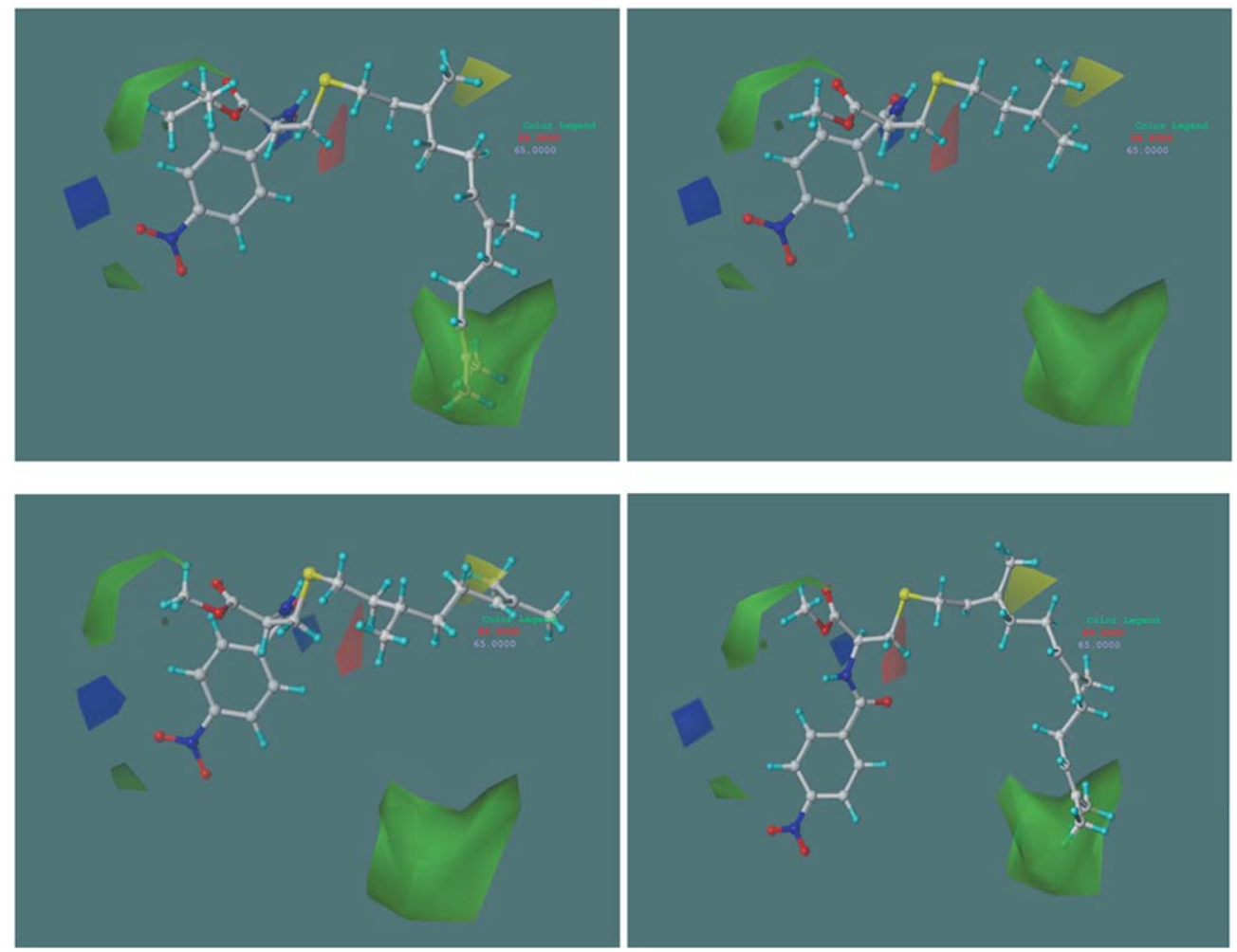

Fig. (7). The contour map with L-75 (top left panel), L-81 (top right panel), L-86 (bottom left panel) and L-70 (bottom right panel) embedded in it.

The resistance of the substrates to hydrolysis by the cholinesterase enzymes is further testimony that the endogenous polyisoprenylated methylated proteins are indeed most likely specifically metabolized by PMPMEase. An unidentified rod outer segment hydrolase which also shows a preference for polyisoprenylated substrates has been reported [12]. The reliance on polyisoprenylation for high affinity is in agreement with previous work in which no other esterase was identified following the enzyme purification with polyisoprenylated substrate-dependent assay screening of the collected fractions [14]. Polyisoprenyl tranferases are highly specific towards the trans,trans-farnesyl and all trans-geranylgeranyl groups [33]. Likewise, the endoprotease and the methyl transferase are dependent on the polyisoprenylation for effective catalysis [26, 28, 34, 35]. The modifications probably serve a protein-protein interaction function [9] as well as recognition elements for the pathway enzymes.

Previous work in which S-adenosyl-L-methioninedependent methylation precipitated a Parkinson's diseaselike effect [36] that was abrogated by trans,trans-farnesyl-Lcysteine analogs [37,38] indicates that aberrations in the pathway may contribute to more clinical conditions than has been reported. The current study validates the enzyme as the likely liver polyisoprenylated protein-metabolizing esterase and provides a platform for the synthesis of specific high affinity inhibitors to further explore the role of the enzyme in physiology and disease as well as lead compounds for pharmaceutical drug development.

\section{CONCLUSION}

With all other parameters constant and varying only the S-substituents, the Michaelis-Menten kinetics results demon- strate that substrates with the all trans-geranylgeranyl and trans,trans-farnesyl have the highest affinities towards PMPMEase. These are the moieties used in the secondary modification of proteins in eukaryotic cells. Furthermore, PMPMEase has the endoplasmic reticulum-retention signal that would co-localize it with PPMTase. These are strong indications that polyisoprenylated proteins are the putative endogenous substrates of the enzyme. The results further demonstrate that inhibitors synthesized with trans,transfarnesyl and all trans-geranylgeranyl groups are more likely to bind to PMPMEase with higher affinity than to such enzymes as the cholinesterases.

\section{ABBREVIATIONS}

\begin{tabular}{|c|c|c|}
\hline AChE & $=$ & Acetylcholinesterase \\
\hline $\mathrm{BChE}$ & $=$ & Butyrylcholinesterase \\
\hline CoMFA & $=$ & Comparative molecular field analysis \\
\hline DCM & $=$ & Dichloromethane \\
\hline EPL & $=$ & $\begin{array}{l}\text { Esterase from porcine liver, also known } \\
\text { as }\end{array}$ \\
\hline PMPMEase & $=$ & $\begin{array}{l}\text { Polyisoprenylated methylated protein } \\
\text { methyl esterase }\end{array}$ \\
\hline HPLC & $=$ & $\begin{array}{l}\text { High performance liquid chromatogra- } \\
\text { phy }\end{array}$ \\
\hline icmt & $=$ & $\begin{array}{l}\text { Isoprenylated carboxylmethyl trans- } \\
\text { ferase }\end{array}$ \\
\hline$k_{\text {cat }}$ & $=$ & Steady state enzyme activity \\
\hline 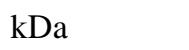 & $=$ & Kilodalton \\
\hline
\end{tabular}




$\begin{array}{lll}K_{\mathrm{M}} & = & \text { Michaelis-Menten constant } \\ \mathrm{NMR} & = & \text { Nuclear magnetic resonance } \\ \mathrm{p} K_{\mathrm{M}} & = & -\log \text { Michaelis-Menten constant (in } \\ & & \text { molar concentration units) } \\ \text { PNB } & = & \text { p-nitrobenzoyl } \\ \text { PPMTase } & \text { Polyisoprenylated protein methyl trans- } \\ \text { QSAR } & = & \text { Quantitative structure-activity relation- } \\ \text { TLC } & = & \text { Thin layer chromatography }\end{array}$

\section{ACKNOWLEDGEMENTS}

This work was supported by NIH/NIGMS/SCORE Grant number GM 08111-35 and by the Pharmaceutical Research Center NIH/NCRR Grant number G12 RR0 3020.

We thank Dr. Leonid Koikov for most of the synthesis, NMR and optical rotation analysis of the substrates.

\section{REFERENCES}

[1] Perez-Sala D. Protein isoprenylation in biology and disease: general overview and perspectives from studies with genetically engineered animals. Front Biosci 2007; 12: 4456-72.

[2] Roskoski R, Jr. Protein prenylation: a pivotal posttranslational process. Biochem Biophys Res Commun 2003; 303:1-7.

[3] Konstantinopoulos PA, Karamouzis MV, Papavassiliou AG. Posttranslational modifications and regulation of the RAS superfamily of GTPases as anticancer targets. Nat Rev Drug Discov 2007; 6: 541-55.

[4] Ghobrial IM, Adjei AA. Inhibitors of the ras oncogene as therapeutic targets. Hematol Oncol Clin North Am 2002; 16: 1065-88.

[5] Tolmachova T, Anders R, Abrink M, et al. Independent degeneration of photoreceptors and retinal pigment epithelium in conditional knockout mouse models of choroideremia. J Clin Invest 2006; 116: 386-94.

[6] Seabra MC, Ho YK, Anant JS. Deficient geranylgeranylation of Ram/Rab27 in choroideremia. J Biol Chem 1995; 270: 24420-27.

[7] Bergo MO, Lieu HD, Gavino BJ, et al. On the physiological importance of endoproteolysis of CAAX proteins: heart-specific RCE1 knockout mice develop a lethal cardiomyopathy. J Biol Chem 2004; 279: 4729-36.

[8] Bergo MO, Leung GK, Ambroziak P, et al. Isoprenylcysteine carboxyl methyltransferase deficiency in mice. J Biol Chem 2001, 276: 5841-45.

[9] Gosser YQ, Nomanbhoy TK, Aghazadeh B, et al. C-terminal binding domain of Rho GDP-dissociation inhibitor directs N-terminal inhibitory peptide to GTPases. Nature 1997; 387: 814-19.

[10] Kloog Y, Cox AD. Prenyl-binding domains: potential targets for Ras inhibitors and anti-cancer drugs. Semin Cancer Biol 2004; 14: 253-61.

[11] Philips MR, Pillinger MH, Staud R, et al. Carboxyl methylation of Ras-related proteins during signal transduction in neutrophils. Science 1993; 259: 977-80.

[12] Tan EW, Rando RR. Identification of an isoprenylated cysteine methyl ester hydrolase activity in bovine rod outer segment membranes. Biochemistry 1992; 31: 5572-78.

[13] Perez-Sala D, Tan EW, Canada FJ, Rando RR. Methylation and demethylation reactions of guanine nucleotide-binding proteins of retinal rod outer segments. Proc Natl Acad Sci USA 1991; 88: 3043-46.

[14] Oboh OT, Lamango NS. Liver prenylated methylated protein methyl esterase is the same enzyme as sus scrofa carboxylesterase. J Biochem Mol Toxicol 2008; 22: 51-62.
[15] Bencharit S, Edwards CC, Morton CL, et al. Multisite promiscuity in the processing of endogenous substrates by human carboxylesterase 1. J Mol Biol 2006; 363: 201-14.

[16] Fleming CD, Edwards CC, Kirby SD, et al. Crystal structures of human carboxylesterase 1 in covalent complexes with the chemical warfare agents soman and tabun. Biochemistry 2007; 46 : 5063-71.

[17] Bencharit S, Morton CL, Xue Y, Potter PM, Redinbo MR. Structural basis of heroin and cocaine metabolism by a promiscuous human drug-processing enzyme. Nat Struct Biol 2003; 10: $349-56$

[18] Bencharit S, Morton CL, Hyatt JL, et al. Crystal structure of human carboxylesterase 1 complexed with the Alzheimer's drug tacrine: from binding promiscuity to selective inhibition. Chem Biol 2003; 10: $341-49$

[19] Gosselin P, Maignan, C, Rouessac F. Stereospecific synthesis of homogeranic and homoneric acids. Synthesis, 1984; 1984: 876-81.

[20] Lange GL, Gottardo, C. Facile conversion of primary and secondary alcohols to alkyl iodides. Synth Comm 1990; 20: 1473-79.

[21] Mori K, Suguro, T, Masuda, S. Stereocontrolled synthesis of all of the possible stereoisomers of 3,11-dimethylnonacosan-2-one and 29-hydroxy-3,11-dimethylnonacosan-2-one: The female sex pheromone of the german cockroach. Tetrahedron 1981; 37: 132940.

[22] Gemperli M, Hofmann W, Rottenberg M. On the Mechanism of Trypsin-Catalyzed Ester-Hydrolysis: Model Experiments on Substrates. Helv Chim Acta 1965; 48: 939-45.

[23] Brown MJ, Milano PD, Lever DC, Epstein WW, Poulter CD. Prenylated Proteins. A convenient synthesis of farnesyl cysteinyl thioethers. J Am Chem Soc 1991; 113: 3176-77.

[24] Lamango NS. Liver prenylated methylated protein methyl esterase is an organophosphate-sensitive enzyme. J Biochem Mol Toxicol 2005; 19: 347-57.

[25] Tan EW, Perez-Sala D, Canada FJ, Rando RR. Identifying the recognition unit for G protein methylation. J Biol Chem 1991; 266: 10719-22.

[26] Perez-Sala D, Gilbert BA, Tan EW, Rando RR. Prenylated protein methyltransferases do not distinguish between farnesylated and geranylgeranylated substrates. Biochem J 1992; 284: 835-40.

[27] De Busser HM, Van Dessel GA, Lagrou AR. Identification of prenylcysteine carboxymethyltransferase in bovine adrenal chromaffin cells. Int J Biochem Cell Biol 2000; 32: 1007-16.

[28] Stephenson RC, Clarke S. Identification of a C-terminal protein carboxyl methyltransferase in rat liver membranes utilizing a synthetic farnesyl cysteine-containing peptide substrate. J Biol Chem 1990; 265: 16248-54.

[29] David L, Guo XJ, Villard C, Moulin A, Puigserver A. Purification and molecular cloning of porcine intestinal glycerol-ester hydrolase--evidence for its identity with carboxylesterase. Eur J Biochem 1998; 257: 142-48

[30] Mori M, Hosokawa M, Ogasawara Y, Tsukada E, Chiba K. cDNA cloning, characterization and stable expression of novel human brain carboxylesterase. FEBS Lett 1999; 458: 17-22.

[31] Munger JS, Shi GP, Mark EA, Chin DT, Gerard C, Chapman HA. A serine esterase released by human alveolar macrophages is closely related to liver microsomal carboxylesterases. J Biol Chem 1991; 266: 18832-38.

[32] Ghosh S. Cholesteryl ester hydrolase in human monocyte/ macrophage: cloning, sequencing, and expression of full-length cDNA. Physiol Genomics 2000; 2: 1-8.

[33] Yokoyama K, Goodwin GW, Ghomashchi F, Glomset JA, Gelb $\mathrm{MH}$. A protein geranylgeranyltransferase from bovine brain: implications for protein prenylation specificity. Proc Natl Acad Sci USA 1991; 88: 5302-06.

[34] Ma YT, Chaudhuri A, Rando RR. Substrate specificity of the isoprenylated protein endoprotease. Biochemistry 1992; 31:11772-77.

[35] Jang GF, Yokoyama K, Gelb MH. A prenylated protein-specific endoprotease in rat liver microsomes that produces a carboxylterminal tripeptide. Biochemistry 1993; 32: 9500-07. 
[36] Lamango NS, Nesby RA, Charlton CG. Quantification of Sadenosylmethionine-induced tremors: a possible tremor model for Parkinson's disease. Pharmacol Biochem Behav 2000; 65: 523-29.

[37] Lamango NS, Ayuk-Takem LT, Nesby R, Zhao WQ, Charlton CG. Inhibition mechanism of S-adenosylmethionine-induced movement deficits by prenylcysteine analogs. Pharmacol Biochem Behav 2003; 76: 433-42.

[38] Lamango NS, Charlton CG. Farnesyl-L-cysteine analogs block SAM-induced Parkinson's disease-like symptoms in rats. Pharmacol Biochem Behav 2000; 66: 841-49.

Received: April 4, 2009

Revised: May 7, 2009

Accepted: May 7, 2009

(c) Nazarius S. Lamango; Licensee Bentham Open

This is an open access article licensed under the terms of the Creative Commons Attribution Non-Commercial License

(http://creativecommons.org/licenses/by-nc/3.0/) which permits unrestricted, non-commercial use, distribution and reproduction in any medium, provided the work is properly cited. 